\title{
Equivalent genetic regulatory networks in different contexts recover contrasting spatial cell patterns that resemble those in Arabidopsis root and leaf epidermis: a dynamic model
}

\author{
MARIANA BENÍTEZ1,\#, CARLOS ESPINOSA-SOTO ${ }^{1, \#, ~ P A B L O ~ P A D I L L A-L O N G O R I A ², ~ J O S E ́ ~ D I ́ A Z ³ ~}$ \\ and ELENA R. ALVAREZ-BUYLLA ${ }^{1, *}$ \\ ${ }^{1}$ Instituto de Ecología and ${ }^{2}$ Instituto de Investigación en Matemáticas Aplicadas y Sistemas, Universidad Nacional Autónoma de México, \\ Mexico, D.F. and ${ }^{3}$ Facultad de Ciencias, UAEM, Cuernavaca, Morelos, Mexico.
}

\begin{abstract}
In Arabidopsis thaliana, leaf and root epidermis hairs exhibit contrasting spatial arrangements even though the genetic networks regulating their respective cell-fate determination have very similar structures and components. We integrated available experimental data for leaf and root hair patterning in dynamic network models which may be reduced to activatorinhibitor models. This integration yielded expected results for these kinds of dynamic models, including striped and dotted cell patterns which are characteristic of root and leaf epidermis, respectively. However, these formal tools have led us to novel insights on current data and to put forward precise hypotheses which can be addressed experimentally. In particular, despite subtle differences in the root and leaf networks, these have equivalent dynamical behaviors. Our simulations also suggest that only when a biasing signal positively affects an activator in the network, the system recovers striped cellular patterns similar to those of root epidermis. We also postulate that cell shape may affect pattern stability in the root. Our results thus support the idea that in this and other cases, contrasting spatial cell patterns and other evolutionary morphogenetic novelties originate from conserved genetic network modules subject to divergent contextual traits.
\end{abstract}

KEY WORDS: gene network, activator-inhibitor, hair patterning

\section{Introduction}

In multicellular organisms cell types are often arranged in a non-random manner, resulting in spatial patterns that may have important functional roles. Cell identity can be acquired and maintained by at least two non-excluding mechanisms, one involving cell-lineage and the other, positional information. The latter seems to be the predominant mechanism in plants (Scheres, 2001). In positional information dependent systems cells attain their identity through mediation of signals (chemical or other) that are not homogenously distributed throughout the organism. Under such circumstances, a pattern is likely to result from the interplay between gene regulatory networks and positional information, that includes contextual traits such as initial conditions, cell arrangement and domain size, geometry and growth (Goodwin, 2001).

Root and leaf epidermal cell patterning in Arabidopsis thaliana
(Arabidopsis hereafter) provides a simple system to explore the processes by which gene regulatory circuits, coupled to the action of contextual conditions, determine spatial cell arrangement in biological systems. In addition, this system has been the subject of careful genetic studies and there is a vast amount of experimental evidence that can be integrated in a dynamic gene network model (see reviews in: Pesch and Hülskamp, 2004; Serna, 2005; Dolan, 2006 and references therein). Leaf epidermis bears hairs (trichomes) interspersed across the leaf surface. These structures tend to appear away from each other (Larkin et al., 1996) resulting in dotted patterns with no clusters (Fig. 1A). Arabidopsis root epidermis has two cell types, hair and non-hair

Abbreviations used in this paper: AC, activator complex; bHLH, basic helixloop-helix; CPC, CAPRICE; EGL3, ENHANCER OF GLABRA3; GL3, GLABRA3; H, hair (cell); NH, non-hair (cell); SCM, SCRAMBLED; TTG, TRANSPARENT TESTAGLABRA;TRY, TRIPTYCHON;WER, WEREWOLF

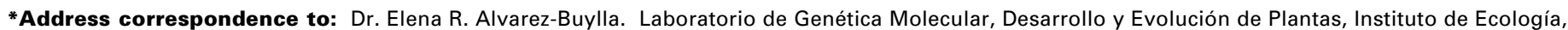

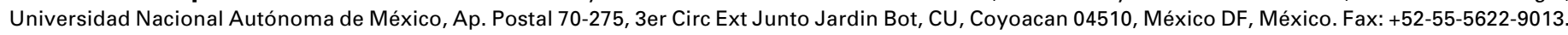
e-mail: elena.alvarezbuylla@gmail.com
}

\# Note: Both authors contributed equally to this work. 

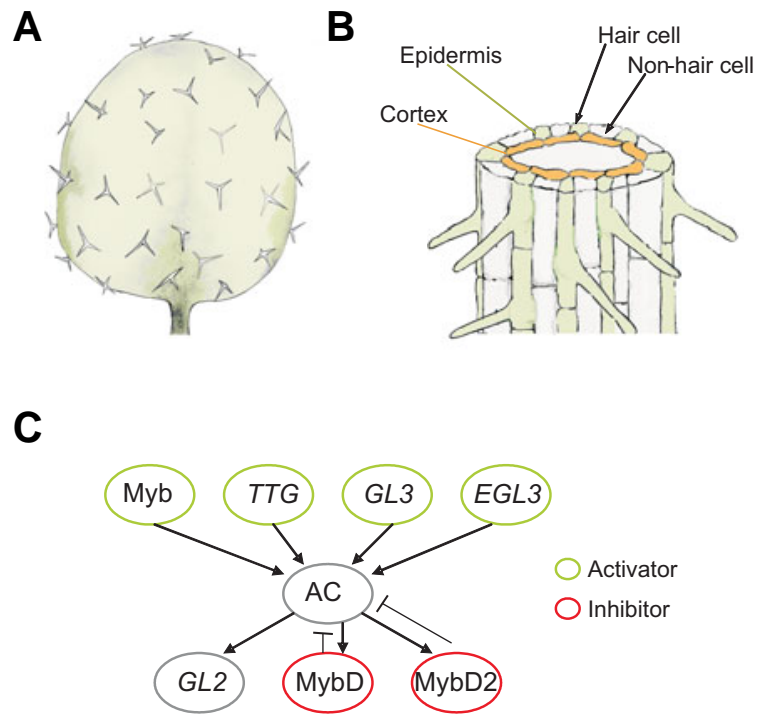

Fig. 1. Networks regulating trichome and root hair patterns in Arabidopsis. (A) Sparsely dotted trichome pattern in a wild type Arabidopsis leaf. (B) Striped trichoblast pattern in Arabidopsis roots. Root hairs develop on epidermal cells that contact two cortical cells. (C) Genes that are not shared between these two networks belong to the same gene families. Myb corresponds to GL1 in leaves and to WER in roots, while MybD corresponds to TRY in leaves and to CPC in roots and MybD2 stands for CPC and TRY in leaves and roots, respectively. CPC and TRY have a partially redundant inhibitory effect on the Activator Complex (AC), these are known as the inhibitory elements of the network. The AC is composed of the activator genes TTG, GL3, EGL3 and GL1 or WER. Drawing (A) based on photograph from Larkin et al. (1996).

cells that develop from trichoblasts and atrichoblasts, respectively. In all Brassicaceae species, including Arabidopsis, the root exhibits an arrangement of alternating cell files composed by either trichoblasts or atrichoblasts (Fig. 1B; Galway et al., 1994; Dolan, 1996).

Despite the different spatial cell patterns found in leaf and root epidermis, the gene networks that underlie cell type subspecification in roots and leaves are very similar (Fig. 1C). Some genes are involved in both the root and leaf systems (Galway et al., 1994; Di Cristina et al., 1996; Payne et al., 2000; Bernhardt et al., 2003) and few are not (Oppenheimer et al., 1991; Lee and Schiefelbein, 1999). However, for each privative gene of one of these systems there is a gene belonging to the same gene family that is privative of the other one. Moreover, interactions among the genes seem to be preserved in both cases (Pesch and Hülskamp, 2004), despite subtle differences (Zhang et al., 2003; Bernhardt et al., 2005; Kurata et al., 2005). Therefore, networks with very similar architectures and molecular components yield strikingly different spatial cell arrangements in leaf and root epidermis (Fig. 1).

The contrasting patterns could be due to differences in the kinetic functions of the networks in each epidermal system (Meinhardt, 1982; Murray,1989). However, experimental data suggest that this is not the case because, at least for two key network components, wild type spatial cell patterns are recovered in transgenic lines that bear constructs with promoters swapped between leaf and root genes (Lee and Schiefelbein, 2001). An alternative explanation would imply that different contextual traits providing positional cues in the tissues are responsible for the distinct spatial patterns of cell types in leaves and roots.

In Arabidopsis roots, only epidermal cells that overlie two cortical cells become trichoblasts (Fig. 1B; Galway et al., 1994), suggesting the presence of a positional signal that biases root epidermal cell fate. Since root cells mainly divide in one direction, all descendants of a root initial are arranged in a row along the baso-apical axis (Meyerowitz, 1997), giving rise to a constant spatial relation among distinct root cell layers. This does not happen in leaves. Such contextual differences may be responsible for the contrasting spatial cell patterns of root and leaf epidermis.

Lee and Schiefelbein (2001) have suggested that genetic networks underlying hair and non-hair determination and arrangement are equivalent in both epidermal systems. However, although these investigators have proven that the coding regions of two genes (WEREWOLF, WER and GLABRA1, GL $)$ are functionally equivalent, the different promoters could still be responsible for different transcription rates that could in turn be critical for attaining the two patterns. It has also been proposed that these networks can be understood as an activator-inhibitor dynamical system, a type of Turing reaction-diffusion system (Pesch and Hülskamp, 2004). Yet, this has not been formally tested.

In this article we first show that despite slight differences, the root and leaf networks are dynamically equivalent and then we explicitly test if, assuming such genetic networks with equal kinetics and regulatory interactions, different contextual traits are sufficient or necessary to yield contrasting spatial cell patterns resembling those observed in leaf and root epidermis. To achieve this, we developed network models grounded on experimental data for gene interactions during epidermal cell fate and then built a single generic network for either of both systems (Fig. 1C).

We found that the gene regulatory network (Fig. 1C) exhibits the same qualitative behavior as a simplified discrete system in which only one activator and one inhibitor are considered. Therefore, we reduced the network model to a type of reaction-diffusion system. Here we report that this continuous system, under different contexts is sufficient to recover the two spatial cellular patterns and that the root striped cellular pattern is only recovered when a positional signal affects the system in a specific manner. Moreover, we were able to reproduce and explain empirical results and provide novel predictions on gene interactions that may be tested experimentally. It would not be feasible to achieve these goals without a dynamical model that integrates the available empirical data.

\section{Experimental Evidence}

TRANSPARENT TESTA GLABRA (TTG) and bHLH proteins (GLABRA3, GL3 and ENHANCER OF GLABRA 3, EGL3) form functional multimers with Myb proteins GLABRA1 (GL1) (Larkin et al., 1997; Payne et al., 2000) and WEREWOLF (WER) (Lee and Schiefelbein, 1999; Bernhardt et al., 2003, 2005) in leaf and root, respectively. Hence we refer to the TTG, GL3, EGL3 and GL1 protein complex as the leaf activator and to the TTG, GL3, EGL3 and WER protein complex as the root activator. Both activators promote transcription of GLABRA2 (GL2, Di Cristina et al., 1996; 
Hung et al., 1998; Szymanski et al., 1998; Lee and Schiefelbein, 1999; Payne et al., 2000; Wada et al., 2002; Costa and Dolan, 2003; Bernhardt et al., 2003, 2005;), whose expression determines leaf trichome cell-fate (Rerie et al., 1994; Szymanski et al., 1998) and root atrichoblast identity (Di Cristina et al., 1996). Therefore, the leaf and root activator complexes, in which GL 1 and WER, respectively, take part (Larkin et al., 1993; Lee and Schiefelbein, 1999), determine trichome cell identity in the leaf and atrichoblast fate in the root.

TRIPTYCHON (TRY) and CAPRICE (CPC) are the main repressors of the leaf and root activators, correspondingly (Schnittger et al., 1999; Lee and Schiefelbein, 2002), although both have partially redundant roles in both root and leaf epidermal systems. ENHANCEROF TRIPTYCHONANDCAPRICE 1(ETC1) has also been proven to play an important role in inhibiting both activator complexes (Kirik et al., 2004). Experimental evidence shows that $C P C$ is upregulated by the root activator (Lee and Schiefelbein, 1999, 2002; Wada et al., 2002; Bernhardt et al., 2003; Costa and Dolan, 2003; Ryu et al., 2005). On the other hand, the highest level of TRYexpression occurs in the cells with maximum GL1 and GL3 expression levels (Larkin et al., 1993; Schellmann et al., 2002; Zhang et al., 2003), suggesting that the leaf activator may also activate this inhibitor.

There is no experimental evidence for GL1 or WER selfactivation (Pesch and Hülskamp, 2004). However, WER and $C P C$ have highest levels of expression in atrichoblasts (Lee and Schiefelbein, 1999; Wada etal., 2002), while GL 1 and TRYpeaks of expression occur in trichome cells (Larkin et al., 1993; Schellmann etal., 2002). As TRYand CPCrepress GL 1 and WER expression, respectively, either direct or indirect self-activation of the activators is needed to overcome the negative regulation of the inhibitors. This should be tested experimentally.

GL3and EGL3 take part in the activator complexes, promoting non-hair cell fate in root and trichome fate in leaf (Payne et al., 2000; Bernhardt et al., 2003; Zhang et al., 2003). In yeast-two hybrid, the GL3 and EGL3 proteins interact with CPC (Zhang et

Fig. 2. Data on bHLH expression and movement (Bernhardt et al., 2005) are consistent with Werewolf (WER) and bHLH proteins being mainly located in the same type of cells. (A) Schematic of a two-cell system. WER represses the production of bHLH proteins, such as GL3 and EGL3, which tend to diffuse from high to low concentrations and can move to neighboring cells. As bHLH proteins interact with WER in a bHLHMER complex, the concentration of free bHLH decreases in nonhair (NH) cells; consequently, bHLH produced in neighboring hair (H) cells keeps moving towards cells where WER is expressed. Since the activator complex seems to negatively regulate the expression of GL3 and EGL3 (Bernhardt et al., 2005), no bHLH protein is actually produced in cells where WER is present. Free GL3 dynamics were modeled according to $\frac{\partial G}{\partial t}=D \nabla^{2} G+\frac{k_{1}}{C+k_{1}}-k_{2} G-k_{3} W G+k_{4} C$ while WER dynamics respond to $\frac{\partial W}{\partial t}=f(x)-k_{5} W+k_{4} C-k_{3} W G$ and the rate of change in the concentration of the GL3-WER complex is given by $\frac{\partial C}{\partial t}=k_{3} W G-k_{4} C$ where $D=0: 35, k_{1}=0: 3, k_{2}=0: 22, k_{3}=0: 6, k_{4}=$ $0: 15, k_{5}=0.2$ and $f(x)$ equals 0.5 in NH cells and 0 in $H$ cells. (B,C) In a continuous system in which the WER/bHLH complex is being formed, the total amount of bHLH protein (free and attached to WER) present in $\mathrm{NH}$ cells is higher than that present in $\mathrm{H}$ cells. al., 2003). Despite being included in the activator complexes, a recent work (Bernhardt et al., 2005) has demonstrated that GL3 and EGL3 are not primarily expressed in non-hair root cells. Surprisingly, they are expressed in the hair cells and move towards those where the other activating elements are mainly expressed. Bernhardt et al. (2005) proposed that GL3and EGL3 are activated by the inhibitor $C P C$ and are repressed by the activator complex. In contrast to these results, in leaves $G L 3$ expression occurs in trichome cells, where GL1 maximum expression and hence maximum levels of leaf activator activity, are found.

Although the above data indicate that root and leaf network topologies are slightly different, it seems that the dynamical behavior of both networks remains qualitatively the same because: i) since other elements (e.g. WER) of the activator complex are present only in non-hair cells and are a limiting factor for the formation of the activator complex, the concentration of such complex is always higher in non-hair cells, ii) diffusion tends to homogenize concentration of free GL3 or EGL3 proteins, iii) CPC,

A

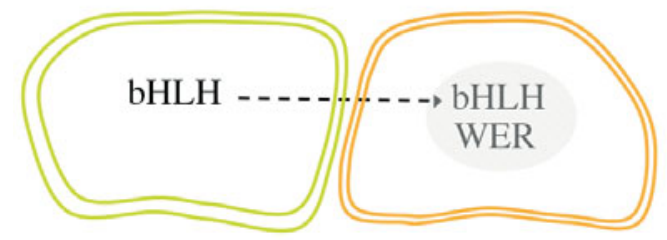

H cell

NH cell

(bHLH production)

(bHLH acumulation)

B

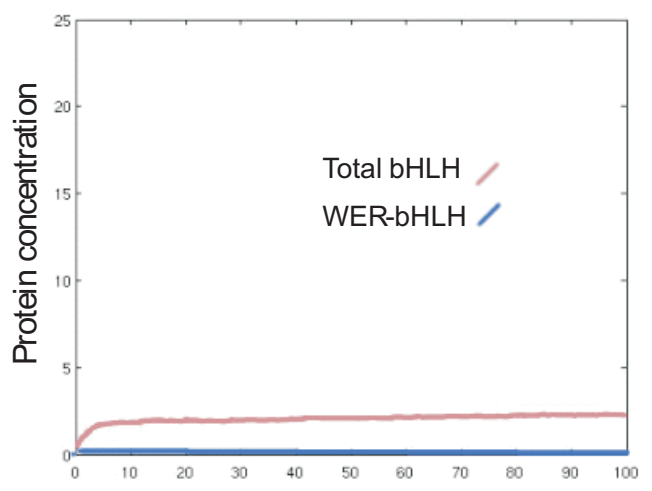

C

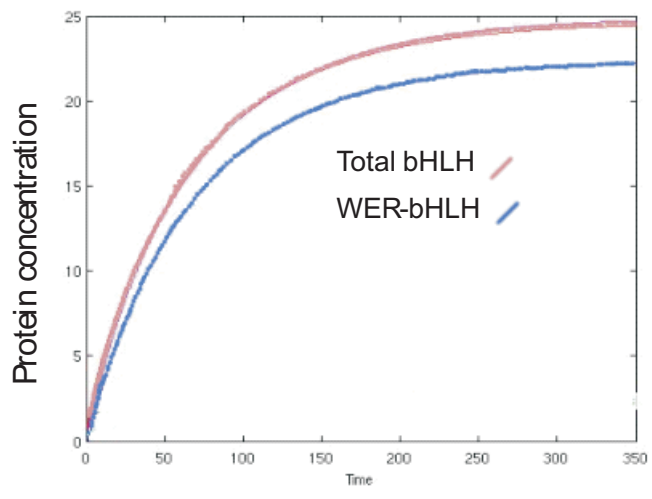

$\mathrm{NH}$

Time
$\mathrm{H}$ 
which interacts with GL3 and EGL3, is expressed in hairless cells and moves to hair cells, exhibiting an homogenous or even atricoblast-biased concentration in root epidermal cells (Wada et al., 2002; Kurata et al., 2005); finally, iv) the total concentration of the GL3 or EGL3 protein is given by free protein plus that attached to the activator complex; then the concentration of total bHLH protein is always higher in the cells where both WER and TTG are present in comparison to cells where these components of the activator complex are not expressed. This final result is equivalent to that given by the leaf network where GL 1 and GL3or EGL3are mainly expressed in the same cell type in leaf epidermis, which could be due to GL3/EGL3activation by GL1. Simulation results (Fig. 2) support and illustrate this claim.

\section{Results}

The discrete cell-autonomous network resembles the reaction term of an activator-inhibitor system

Based on experimental evidence summarized above a gene network model was proposed. The network nodes represent genes and protein complexes involved in epidermal cell type determination and the edges stand for interactions among them (see Fig. 1 and Methods for a more detailed description of this model). Such interactions were formalized in logical rules that enabled a dynamic analysis of the network.

We considered all possible initial conditions in the network and found only two steady states representing self-sustained gene activity profiles (see Appendix).
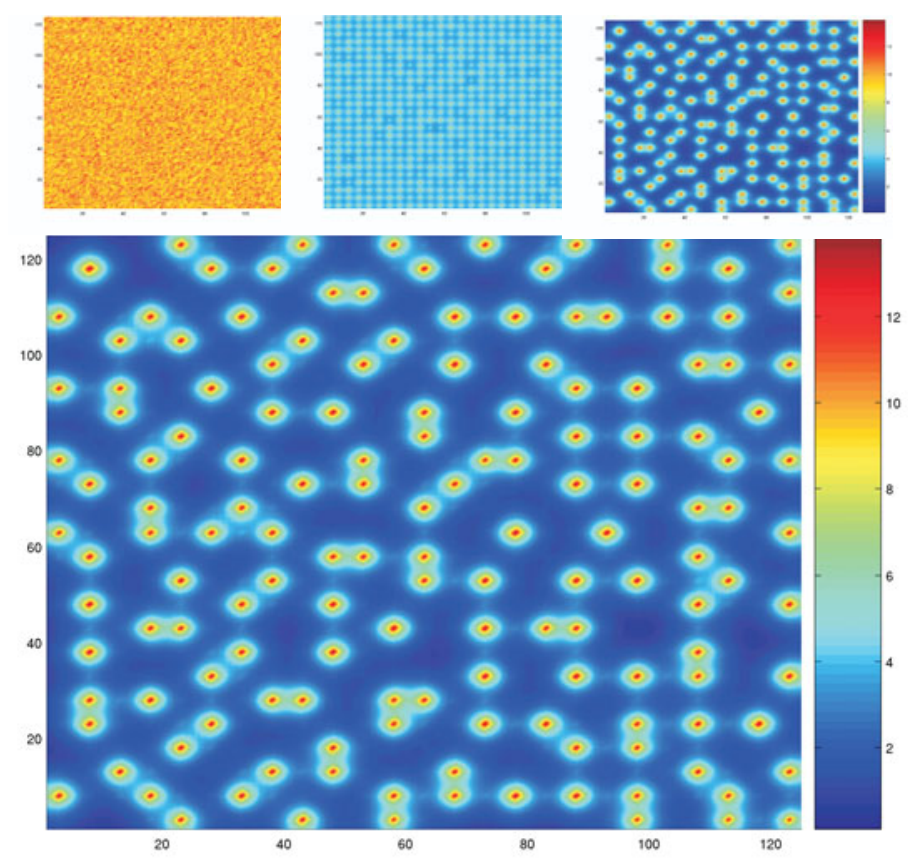

Fig. 3. Activator:inhibitor ratio for the leaf system at different time steps. Attainment of stable patterns for the leaf system. Nuclei are arranged in a regular lattice. Time periods for all small panels from left to right: $t=0, t=10$ and $t=100$. The large panel corresponds to $t=2000$. Parameter values for all simulations are: $\mathrm{k}_{1}=0.3 ; \mathrm{k}_{2}=0.3 ; \mathrm{k}_{3}=0.4 ; \mathrm{k}_{4}=0.75$. The initial diffusion rates are: $D_{a}=0.15 ; D_{i}=1.65$. Red represents high values of the activator:inhibitor concentration ratio.
Kauffman suggested that steady states, or attractors, of gene networks correspond to cell types (Kauffman, 1969, 1996) and this proposal has been explored with the use of some well documented discrete gene networks grounded on experimental data (Mendoza and Alvarez-Buylla, 1998, 2000; Mendoza et al., 1999; Albert and Othmer, 2003; Espinosa-Soto et al., 2004). Congruent with this hypothesis, the configuration of the nodes' states for the two attractors matches the expression profiles of the two cell types in Arabidopsis epidermis. In one of these attractors the inhibitors, but also the activator complex and therefore $G L 2$, are not active while in the other, they all are (see Appendix). The configuration with GL2'off' corresponds to non-trichome cells in the leaf and to trichoblasts in the root.

We introduced a reduced network system that maintains the relevant traits of the complete network dynamics. The reduced network has only two elements: an activator that positively regulates an inhibitor and itself and the inhibitor, which in turn, negatively regulates the activator. The activator stands for the activator complex (integrating the activity of WER or GL1, GL3, $E G L 3$ and TTG) and the inhibitor represents the joint action of $T R Y$ and $C P C$. The dynamical analysis of this system led to similar results as those obtained for the whole network: it has only two attractors with gene activation configurations matching those observed in either of the two cell types.

In addition, the comparison of the states diagram of the discrete reduced system and the well-known phase plane associated to the reactive terms of a typical activator-inhibitor continuous system, strongly suggests that these are qualitatively equivalent. They both have two steady states (network system) or equilibrium points (continuous activator-inhibitor system) and exhibit comparable trajectories (see Appendix). Hence, the reduction of the complete network to an activator-inhibitor system is justified. By doing this we propose that the cell-autonomous gene network can be compared to the reactive terms of a continuous activator-inhibitor system, while the spatially explicit terms of the latter may account for positional biases and cell-cell communication via the inhibitors movement. We intend to represent a system in which positional information and cell communication affect the initial conditions of dynamic gene networks that ultimately attain a steady state corresponding to a cell type.

\section{A leaf activator-inhibitor system recovers spatial patterns that resemble those observed for trichomes}

Turing (1952) proposed systems in which reactants with different diffusion coefficients can produce spatial patterns de novo and maintain them. Activator-inhibitor systems are a particular case of reaction-diffusion (Turing) systems and are based on local self-activation and lateral inhibition (Meinhardt, 1982; Nijhout, 2003). Given the results obtained with the discrete system, we introduced a spatially explicit model of this kind. In it two interactors are considered: the activator and the inhibitor, which correspond, in the discrete system, to the AC node (WER/GL1, TTG, GL3, EGL3) and to the partially redundant action of $C P C$ and $T R Y$, respectively. The activator promotes its own synthesis and that of the inhibitor. The inhibitor, in turn, represses the synthesis of the activator complex (see equations and a more detailed description of this model in Methods).

Even though it is known that activator-inhibitor systems can produce qualitatively different patterns when subject to different 
A

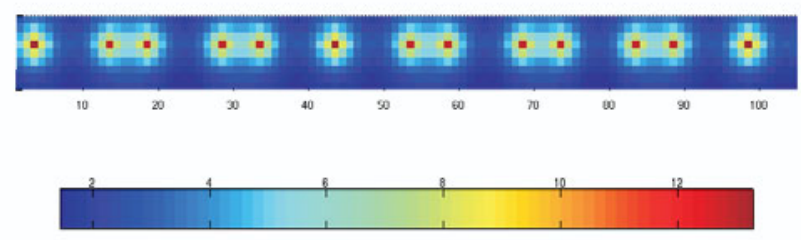

C

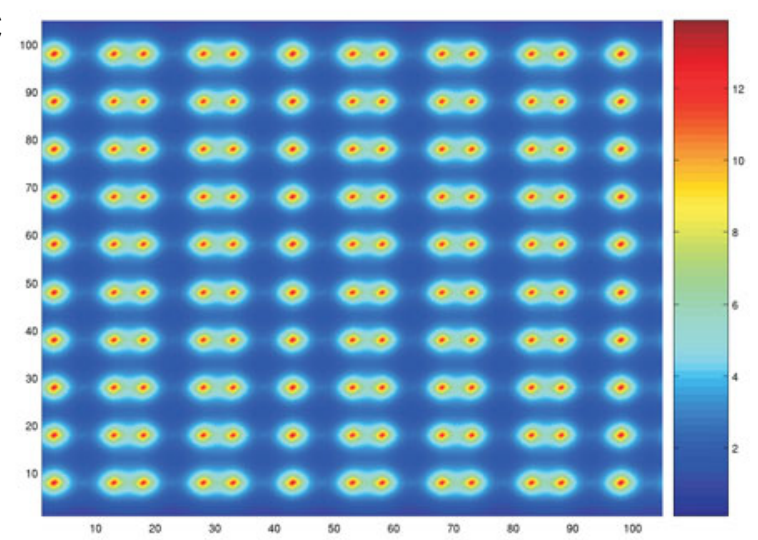

B

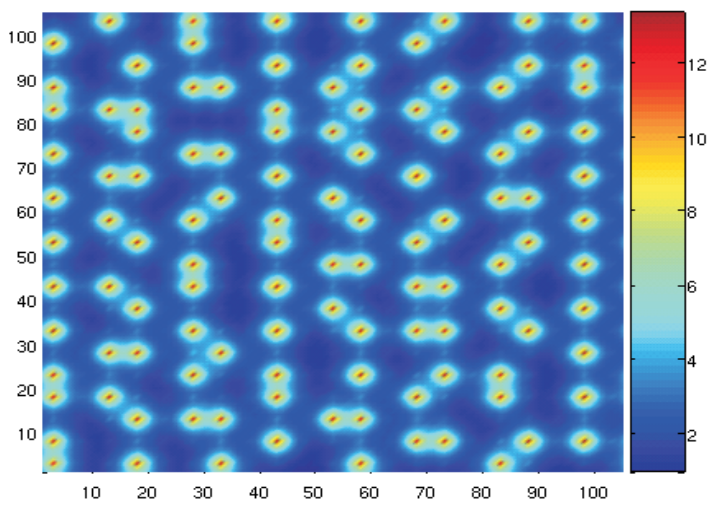

D

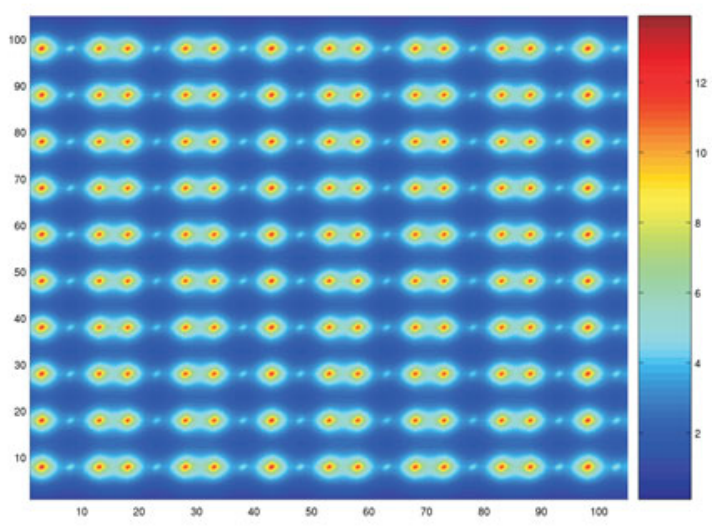

Fig. 4. Patterns for the root system. (See Equations 3 and 4 in the Methods section). (A) Stable pattern generated in a one-dimensional ring of cells (B) Spatially unstable root-like patterns observed for the two-dimensional root system (Equations 3 and 4 in the Methods section) considering squared cells. Notice simulated hair cell bands are recognizable but somewhat unaligned. (C) Here, nearest neighbor of a nucleus on the $x$ axis is closer to it than its nearest neighbor on the y axis and hence little variation is observed in the activator:inhibitor ratio within the same column. (D) The 35S:WER root system also yields striped patterns. W=0.4 stands for the contribution of WEREWOLF protein due to the 35S promoter (see Equation 5 in the Methods section). In all cases, time stable patterns at $t=2000$ are shown, parameter values for all simulations as in Fig. 3 and $f(x)=0.06$ and is included in the system as in Equation 3 (Methods section). Red represents high values of the activator:inhibitor concentration ratio.

parameter values, we address here if an activator-inhibitor system can produce patterns similar to those observed in leaf and root epidermis with the same parameter values but under contrasting contextual conditions.

For the leaf system, we considered a squared spatial domain and zero-flux boundary conditions, representing a leaf's section. The system (equations (1)and (2)in Methods) was initialized with random conditions for the concentration of the leaf activator and a constant value for that of the inhibitor, therefore, the activator:inhibitor ratio could never be undefined. We picked parameter values in the Turing space and as expected for systems of this kind, the simulations for the leaf equations recovered dotted patterns. Figure 3 shows a color-coded graph of the steady ratio of leaf activator over leaf inhibitor throughout the spatial domain.

The root model recovers Arabidopsis-like root-hair patterns and predicts the way the scrambled gene affects the network

In our model, contextual differences between the two epidermal systems are considered. The most important one concerns the fact that only root epidermal cells that overlie two cortical cells develop as trichoblasts, whereas the rest develop as atrichoblasts (Galway et al., 1994). This suggests the existence of a signal from the cortex that biases epidermal cell identity. The signal from the cortex seems to be constant throughout the process of epidermal cell differentiation because when occasional longitudinal anticlinal divisions occur in root epidermal cells, daughter cells attain their identity according to their position relative to cortical cells (Berger et al., 1998). This kind of signal has not been found in the leaf epidermis. Hence, we introduced an additional constant term into one of the root system's equations, representing positional cues produced by the signal from the cortex cells (equations 3 and 4 in Methods). The signal has been shown to partially depend on the gene SCRAMBLED (SCM), which encodes a membrane kinase receptor protein (Kwak et al., 2005). In principle, this signal could act either positively or negatively on the inhibitor or the activator. We tested all four possibilities (see Fig. 5 and Discussion) of which, within a wide range of signal values (0.06 to 0.30$)$, only the one assuming a positive regulation over the activator yielded the expected striped spatial pattern typical of Arabidopsis 
A

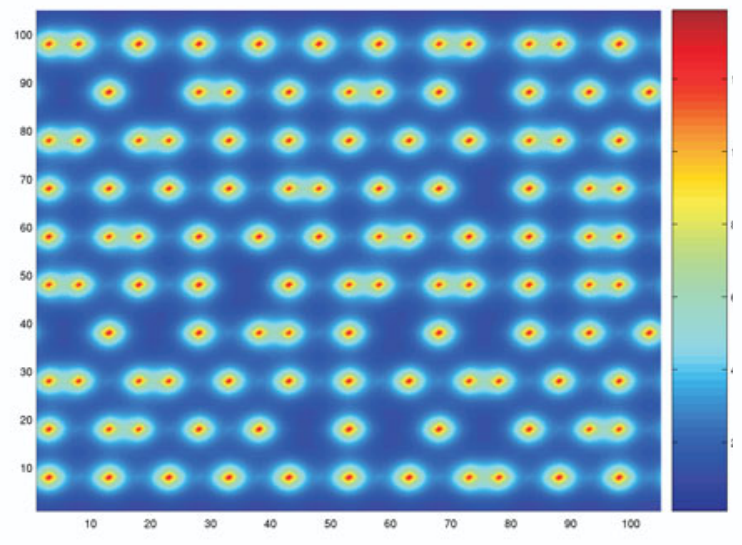

C

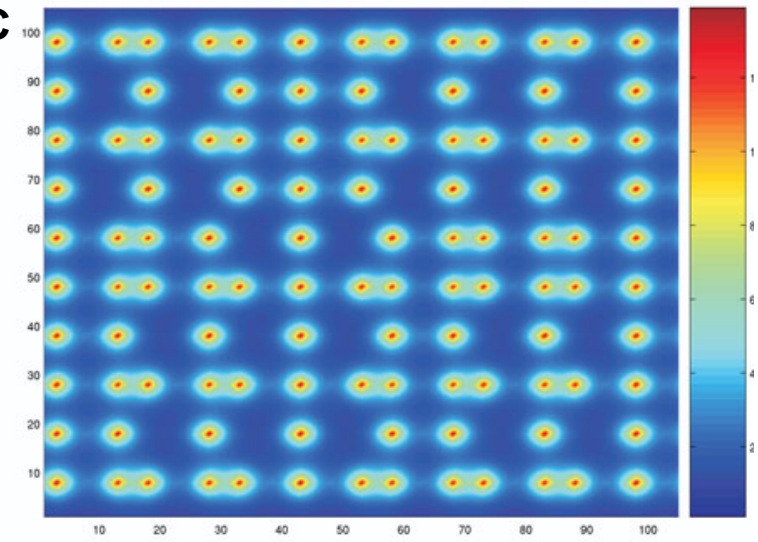

B

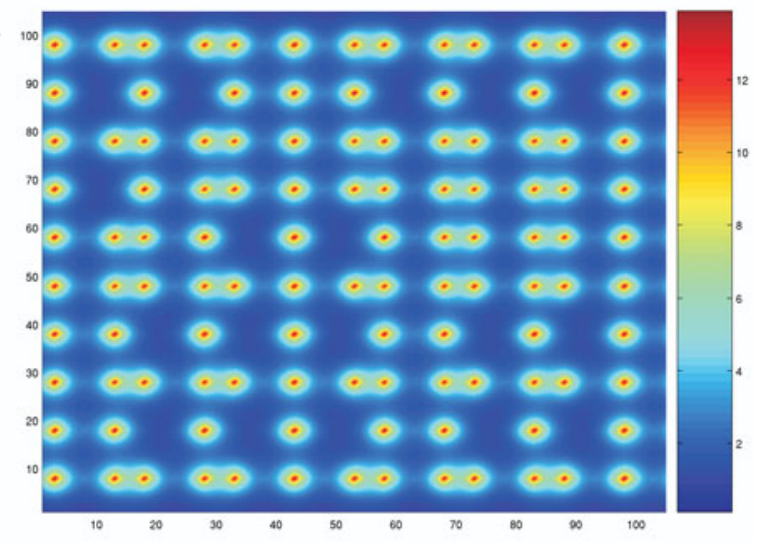

D

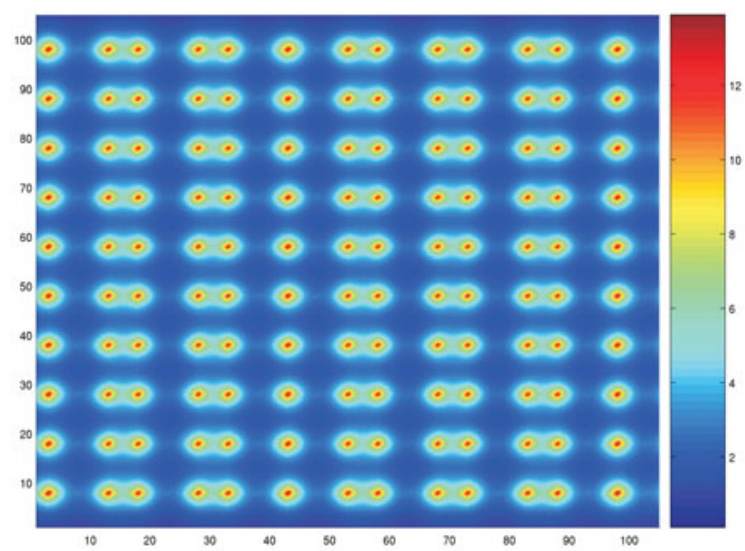

Fig. 5. Root-like patterns in the model with elongated cells are spatially stable only when the signal corresponding to that going from cortical to epidermal cells is assumed to positively regulate the activator (D). The root pattern is not recovered when the signa/(A) represses the inhibitor, (B) represses the activator or (C) activates the inhibitor. Initial conditions for each simulation were random and the parameters were the same as in Fig. 3. Red represents high values of the activator:inhibitor concentration ratio.

root epidermis. Therefore, we present the results for this particular type of signal (equations 3 and 4 in Methods).

When considering squared root cells in a two-dimensional domain, the resulting patterns present some bands that resemble observed hair cell stripes; however, the concentration maxima are not aligned as clearly as in real roots (Fig. 4B). Therefore the obtained patterns can be described as spatially unstable root patterns. There are a number of factors that could yield the formation of almost perfect cell type bands in actual roots. For instance, the intensity of the cortex signal, the effect of unknown genetic elements or interactions in the network or the curvature of the domain.

Another possibility is that cell geometry affects the final twodimensional patterns. Cell elongation, which actually takes place in the elongation zone basal to the root meristem, may affect final pattern formation by increasing the distance among nuclei in the root axis direction. Thus, the cortical signal might prevail on the activator-inhibitor mechanism in this direction. This does not undermine the importance of the activator-inhibitor mechanism; it still would be relevant for stripe generation because of it's laterally inhibitory effect on one direction, the one in which nuclei are closer.

We considered a squared domain and zero-flux boundary conditions on two of the borders and periodic boundary conditions on the other two, simulating a cylinder. This was also initialized with random and constant conditions for the concentration of the activator and inhibitor, respectively. If root cells are considered squared, the patterns obtained resemble a striped pattern, yet such patterns are not as clear as those found in real roots (Fig. 4B).

Then, we explicitly test if once the pattern is formed in one dimension, cell elongation can be responsible for pattern spatial stabilization in the two-dimensional domain. To this end, we introduced a two-dimensional root model as the one described by equations (3) and (4) (Methods), but with cells elongated in the baso-apical direction (its nearest neighbor along the $x$-axis is closer than its nearest neighbor along the $y$-axis), similar to those observed in the differentiation root zone. Although root patterning begins before cells are elongated, we postulate that the final striped arrangement in the two-dimensional domain may be stabilized during cell elongation.

Once the root system was specified with elongated cells, we tested all four possible kinds of positional signal that might represent the $S C M$-dependent cue. Given a relatively weak signal intensity (0.06), the clear pattern observed in roots is only recovered when such signal acts as a positive regulator of the root 
activator (Fig. 5). The way in which SCM affects epidermal cell identity was pointed out as one of the key issues that remain to be clarified in order to understand pattern formation in root epidermis (Dolan, 2006). This issue has been explored experimentally, yet empirical data do not allow to discern the necessary and sufficient conditions in particular concerning the magnitude and type of signal, that may recover the observed patterns (Kwak et al. 2005). The type of dynamic system presented here, enable predictions that cannot be based solely on experimental data and thus provide novel dynamical insights on this problem of pattern formation (see Discussion).

Since the root pattern does not arise in a sheet of undifferentiated cells but in a ring of small cells, we also performed simulations of the system described in equations (3) and (4) in a one-dimensional domain with periodic boundary conditions, simulating a ring of cells. We found patterns like those observed in rings of root cells (Fig. 4A). Arrangements obtained for twodimensional systems (equations 3 and 4 in Methods) with squared and rectangular cells can be described as repetitions of the ring patterns, although the system that includes elongated cells exhibits clearer patterns, suggesting that cell elongation or some other stabilizing factor with a similar effect might underlie the emergence of aligned cell type bands.

These results for the root system show that the gene networks responsible for hair patterning in leaves and roots may be equivalent and still able to yield different spatial patterns due to differences in the cellular contexts in which they are embedded.

In order to validate the model we tested if it recovers observed patterns when gene expression is altered. Arabidopsis plants constitutively over-expressing WER(35S:WER)in wild type background show wild type trichoblast and GL2 expression patterns, while werloss of function homozygous mutants that overexpress WER present ectopic GL2 expression (Lee and Schiefelbein, 2002). Available experimental data does not provide a clear explanation of how the WER endogenous copy can buffer this increase in WER expression. To address this issue and further test the model we simulated this experiment by assuming a constitutive production of the activator in the root system and by modifying the root activator (equation 5 in Methods). As it happens in 35S:WER plants, columns in which the ratio of activator:inhibitor is higher are still formed, reinforcing the compatibility of our model with experimental data (Fig. 4D). This result suggests that 35S:WER-dependent inhibitor upregulation preserves the activator:inhibitor ratio. Under this scenario, the pattern is recovered because of spatial differences in endogenous WER expression.

It is noteworthy that both the leaf and root patterns are robust to strong noisy perturbations (see Appendix). This suggests that the system is able to buffer developmental noise and canalizes the patterns to those expected in a deterministic system. The system's parameters may be associated to genetic modifications affecting production, diffusion or degradation rates of the molecules involved. By randomly varying parameter values, we found that the patterns obtained do not depend on particular or rare combinations of parameters. Both systems are considerably robust to alterations in parameter values, although the root system with elongated cells is more sensitive than the leaf one (see Appendix).

Interestingly, analyses presented in the Appendix also support that it is not likely that a system like the one presented in (1) and (2) (Methods), without a biasing signal, is able to render striped root-like patterns.

\section{Discussion}

Development of morphological traits depends not only on complex interactions among an organism's molecular constituents but also on specific properties of the domain where morphogenesis occurs. The complexity of such processes and the overwhelming amount of data that is rapidly accumulating is demanding the use of formal dynamical models that integrate available information. These models provide a solid base to understand biological developmental systems, as they suggest hypotheses that can be addressed experimentally, provide tools to interpret counterintuitive experimental data and enable the performance of vast computer explorations on parameter values or initial conditions that can then be tested in vivo.

Trichoblast and trichome pattern formation in Arabidopsis has been attributed to an activator-inhibitor mechanism (Pesch and Hülskamp, 2004). Here we provide a dynamic model that supports this. All relevant properties required for such a mechanism are preserved in our discrete network model that integrates most of the genes known to participate in these processes and in a reduced version of it. The reduced network model is dynamically very similar to the well-mixed case (no diffusion) of a typical activator-inhibitor system. It is important to stress that the steady states of the discrete network systems are compared to the equilibrium points of the reactive terms of the activator-inhibitor continuous system, suggesting that attractors or steady states of gene networks correspond to different cell types and that contextual traits (including positional information) affect the initial conditions and trajectories that lead to such attractors.

Experimental data had already suggested that equivalent networks could underlie leaf and root epidermal cell-fate determination (Lee and Schiefelbein, 2001). However, recent evidence suggested differences in the regulation of GL3 and EGL3 (Bernhardt et al., 2005). We have shown here that despite these dissimilarities, the root and leaf genetic networks behave qualitatively the same (Fig. 2).

Indeed, the model grounded on experimental data that we put forward has enabled us to formally show that contextual traits are sufficient to yield the contrasting Arabidopsis leaf and root epidermal cell patterns, when equivalent networks are assumed. These contextual conditions might be necessary for stabilizing such patterns. The contextual traits considered were the relative position of epidermal cells with respect to cortex cells - which in the root epidermis determine a biasing signal - and cell shape, that was assumed to be rectangular in the root case, but squared in the leaf system. Cell shape is only one of the possible traits that could stabilize the striped pattern of root epidermis, however our simulations support that cell elongation or a mechanism with a similar effect on the system may indeed contribute to spatially stabilize the two-dimensional patterns.

Further experimental data and computational analyses are needed to test if the gene networks underlying trichome and roothair cell determination are really equivalent. For example, genes that are not active in both networks may have different activation functions or behave differently in terms of transport. If the two 
networks were not equivalent, quantitative parameter differences could be responsible for the attainment of the divergent spatial cellular patterns. However, the stability analysis presented here (see Appendix) suggest that this is not the case, given that the structure of the equations that govern the gene network dynamics is conserved in both systems. Since such analysis leads to the conclusion that for this particular system, stripes are very unlikely to form without a biasing signal, our results suggest that the positional cue is necessary to recover the root spatial pattern.

In this work, we provide new hypotheses that can be addressed experimentally. The first one concerns the nature of the signal associated to SCM. This outstanding issue for the study of pattern formation in epidermis remains unclear (Dolan, 2006). Kwak et al. (2005) suggested that the positional cue mediated by the membrane receptor SCM could act on WER. Still, their results are equally compatible with SCM acting on $C P C$. In scm mutants the same researchers found altered patterns of expression of $C P C, G L 2$ and $W E R$ and suggested that since WER regulates the expression of $G L 2$ and $C P C$, the signal could act exclusively on WER. However, CPC also regulates the expression of GL2 (Wada et al., 2002, 1997; Costa and Dolan, 2003) and WER (Lee and Schiefelbein, 2002), so their data does not discard the possibility that the signal acts exclusively on $C P C$.

The signal associated to the position of epidermal cells relative to cortex cells could act either on the inhibitor or the activator, either favoring or limiting their expression. Interestingly our simulations strongly suggest that assuming equal and relatively small signal intensities, such biasing signal necessarily acts positively on WER or any other component of the activator complex in order to recover the striped pattern of roothair cells. Since the signal was modeled explicitly in the four cases by including the $f(x)$ term with different signs in both the activator and inhibitor equations, the striped pattern can not be an artifact derived from the inclusion of $f(x)$. The model may be modified in order to test alternative hypotheses concerning the nature of the biasing signal and could guide future experiments.

Experimental evidence also supports that the biasing signal in the root acts positively on the activator complex. Plants that overexpress WER but preserve the wild type copy of this gene exhibit normal root-hair and GL2 expression patterns. This does not happen in transgenic plants over-expressing WER with non-functional endogenous $W E R$ (Lee and Schiefelbein, 2002), showing that in order to attain normal trichoblast patterns the functional wild type WER promoter is required. Also, Bünning found that in radish epidermal cells developing independently from other tissues bear root hairs (reviewed in Dolan et al., 1994). These epidermal cells would be deprived from molecular signals coming from the cortex and attain the same cell fate that Arabidopsis root cells have when there is no activator.

The second hypothesis we put forward states that root cell elongation along the baso-apical axis stabilizes the root striped pattern. This hypothesis could be tested by crossing a GL2:GUS or GFP marker line to mutants or pharmacologically treated plants that have altered cell shape (see for example Fagard et al., 2000; Le etal., 2001; Ramirez-Parra etal., 2004; Campanoni and Nick, 2005).

Given that most kinetic parameter estimates are not known, we reduced the root-hair and trichome systems to an analogous system with only two elements. However, we did so based on qualitative equivalence of key dynamical traits of the gene network and the discrete version of the activator-inhibitor system. It should also be noticed that the reduction accurately summarizes most available experimental evidence (see Methods). However, a few factors were left out, such as genes that seem to be upstream from the ones in these networks, for instance REDUCED TRICHOMENUMBER (RTN; Larkin et al., 1996) and GLABROUS INFLORESCENCE STEMS(Gan et al., 2006).

The plant hormones auxin and ethylene also participate in the development of Arabidopsis root hairs (Dolan et al., 1994; Masucci and Schiefelbein, 1996), but they act either downstream or in parallel of the genes included in this study (Masucci and Schiefelbein, 1996; Mendoza and Alvarez-Buylla, 2000) and consequently do not affect the behavior of the networks we worked with, further supporting their dismissal for the specific aims of this contribution. Nevertheless, future models that incorporate all of these factors will be very useful for predicting the relative importance of each gene or molecule in response to different signaling pathways in root-hair and trichome pattern formation and evolution.

Epidermal cell type determination network constitutes a small regulatory module and in this work we address the particular issue of how such gene network and contextual traits give rise to cell type patterns. However, the study of this specific and well documented system provides results that can be discussed in terms of evolution of morphogenesis.

As previously discussed, the root and leaf networks seem to be dynamically equivalent. This finding, along with the fact that an activator-inhibitor mechanism coupled to contextual traits recovers characteristic features of cell patterning, supports the claim that few generic and relatively simple mechanisms could be responsible for generating and maintaining heterogeneities during morphogenesis (Turing, 1952; Meinhardt, 1982).

In order to understand how gene networks take part in the generation of robust cellular patterns it will certainly be useful to characterize pattern-generating regulatory modules in well documented gene networks and point them as potentially generic patterning mechanisms. Congruent with the proposal stated by Pesch and Hülskamp (2004), we identify an activatorinhibitor system that arises from the collective behavior of a gene network and point to this mechanism as a potentially generic one. We emphasize that simple conserved mechanisms, such as the activator-inhibitor, may rely not only on couples of elusive morphogenes, but also on the interactions among several genes.

Our simulations also suggest that the networks underlying root and leaf epidermis cell patterning are robust modules that regulate cell-fate determination in different cellular contexts. Such contexts may have evolved yielding the contrasting spatial patterns found in roots and leaves. If we extrapolate this example to thinking about evolution of pattern formation, we may postulate that equivalent networks might render different patterns due to the evolution of dissimilarities in contextual traits between structures and organisms. This suggests that the alteration of genes associated to key contextual traits could underlie the origin of novel and diverse patterns, even if gene 
regulatory networks of cell fate determination remain qualitatively the same, thereby constituting developmental and evolutionary robust modules (von Dassow et al., 2000). Further analyses are required to test how frequent context-dependent evolution could have been in the history of life.

\section{Methods}

\section{Discrete gene regulatory network dynamics}

Grounded on the experimental data summarized in the Introduction, we defined a network in which most nodes correspond to genes and one corresponds to the 'Activator Complex' (AC) node and represents a protein complex constituted by the products of WER or GL1, GLABRA3 (GL3), ENHANCER OF GLABRA3 (EGL3) and TRANSPARENT TESTA GLABRA (TTG). Edges stand for regulatory interactions between nodes (activation or repression; Fig. 1C). Since this system is restrained to the cell-autonomous ambit, space and protein movement are neglected. In order to enable different activity thresholds when there is experimental information suggesting their existence (Thomas, 1991), some nodes GL 1, WER, CAPRICE (CPC), TRIPTYCHON (TRY), GL3 and AC-can attain three states ( 0 corresponding to no expression, 1 mild expression and 2 high expression), whereas the activity of two nodes (TTG and $E G L 3$ ) is defined by two states (0 no expression, 1 expression).

The experimentally documented regulatory interactions among the nodes were formalized as logical rules (see example in Appendix). Given these, it is possible to follow the dynamics of the network for any initial configuration of the nodes expression states and find if the network attains steady states representing self-sustained gene activity profiles, as those thought to characterize cell-types (Kauffman, 1969, 1996). For simplicity, we used synchronic updating of the nodes' states.

In order to compare trajectories in the discrete system with the vector field of the reaction terms in a typical activator-inhibitor system, we also performed simulations using a reduced system where only two nodes were kept, one representing the activator complex and the other the inhibitor complex.

\section{The activator-inhibitor system}

We introduced a continuous activator-inhibitor system in which an activator element, representing the activator complex, positively regulates itself and an inhibitor element, that represents the joint action of inhibitory genes, $C P C$ and $T R Y$. The inhibitor negatively regulates the activator. These interactions are compatible with the available empirical information summarized in the introduction.

For an activator-inhibitor system to create a pattern it is necessary that the inhibitor moves along a wider spatial range than the activator (Meinhardt, 1982). Experimental evidence suggests that this is the case for both systems, since the activators seem to act locally, while the inhibitors do not. In cpc loss of function mutants the cells that overlie two cortical cells develop as atrichoblasts, instead of trichoblasts as it occurs in wild type plants (Wada et al., 1997). Besides, CPC mRNA is localized only in epidermal cells located on top of a single cortical cell, but the CPC protein is found throughout the root epidermis as assayed by green fluorescent protein, strongly suggesting that the CPC protein moves among cells (Wada et al., 2002). Moreover, the CPC coding sequence bears specific motifs that allow it to move (Kurata et al., 2005). Even though the TRY sequence does not have these motifs (Kurata et al., 2005), it is expressed in trichome cells, but cells that normally do not develop trichomes will develop them when TRY is absent, suggesting that this gene function is also non-cell autonomous (Schnittger et al., 1999). Diffusion is the simplest mechanism of protein movement and it is an important type of intercellular communication during plant development (Kim and Zambryski, 2005). Indeed, the CPC and TRY proteins are much smaller than components of the activator complex (Oppenheimer et al., 1991; Wada et al., 1997; Lee and Schiefelbein, 1999; Schellmann et al., 2002), further suggesting that CPC and TRY may move through plasmodesmata.

Genetic regulation can take place at many levels. For simplicity, we neglected translation, in other words, we assumed transcription (mRNA synthesis) to be the rate-limiting step for a protein to exert its function. Although proteins are synthesized outside the nucleus, transcription regulation requires proteins to bind the DNA and the concentration of the modeled proteins' is evaluated by the promoter, in the nucleus. Hence, we considered that gene activation does not occur throughout the entire spatial domain but only in discrete small areas $(\mathrm{N})$. We arranged these in a regular lattice throughout the whole space. As usual, degradation rate was assumed proportional to concentration. Therefore, we considered the following equations for the rate of activator and inhibitor concentration, respectively:

$$
\begin{aligned}
& \frac{\partial a_{x, y}}{\partial t}=\left\{\begin{array}{lr}
D_{a} \nabla^{2} a_{x, y}-k_{1} a_{x, y}+k_{2} \frac{a_{x, y}{ }^{2}}{i_{x, y}} & \text { if }(x, y) \in N \\
D_{a} \nabla^{2} a_{x, y}-k_{1} a_{x, y} & \text { if }(x, y) \notin N
\end{array}\right. \\
& \frac{\partial i_{x, y}}{\partial t}= \begin{cases}D_{i} \nabla^{2} i_{x, y}-k_{3} i_{x, y}+k_{4} a_{x, y}{ }^{2} & \text { if }(x, y) \in N \\
D_{i} \nabla^{2} i_{x, y}-k_{3} i_{x, y} & \text { if }(x, y) \notin N\end{cases}
\end{aligned}
$$

where trepresents time, $x$ and $y$ the spatial coordinates, a the concentration of the activator, $i$ that of the inhibitor and $\nabla a$ and $\nabla i$ are the laplacians for the activator and inhibitor, respectively, that define diffusive movement of the molecules. The strength of such movement is characterized by the diffusion coefficients $D a$ and $D i$, for the activator and the inhibitor respectively (with $D a \ll D i$ ). $k_{1}$ and $k_{3}$ are degradation constants for the activator and inhibitor, respectively and $k_{2}$ and $k_{4}$ are their corresponding production constants.

For the root system, we introduced a bias representing the signal associated to SCM. We tried positive or negative signals either over the activator or the inhibitor complexes. We found that of the four possibilities, given a relatively small signal ( 0.06 to 0.30 ), only a positive signal over the activator complex was able to recover the observed root-like pattern. Therefore, given the specification of the activator-inhibitor dynamic system presented here, such signal is thus necessary and sufficient to recover the observed pattern and we show here the equations for this case:

$\frac{\partial a_{x, y}}{\partial t}= \begin{cases}D_{a} \nabla^{2} a_{x, y}-k_{1} a_{x, y}+k_{2} \frac{a_{x, y}{ }^{2}}{i_{x, y}}+f(x) & \text { if }(x, y) \in N \\ D_{a} \nabla^{2} a_{x, y}-k_{1} a_{x, y} & \text { if }(x, y) \notin N\end{cases}$

where $f(x)$ represents the positional cues coming from the cortex. $f(x)$ acquires the value of a small positive constant if $x^{\prime}$ is equal to one value of an a priori defined set that corresponds to the nucleus, otherwise $f(x)$ equals zero. The equation for the root inhibitor is the same as that for the leaf inhibitor and all common terms are as in the leaf equations above:

$$
\frac{\partial i_{x, y}}{\partial t}= \begin{cases}D_{i} \nabla^{2} i_{x, y}-k_{3} i_{x, y}+k_{4} a_{x, y}{ }^{2} & \text { if }(x, y) \in N \\ D_{i} \nabla^{2} i_{x, y}-k_{3} i_{x, y} & \text { if }(x, y) \notin N\end{cases}
$$

Non-linear terms are needed in the reaction part of the reactiondiffusion equations to attain pattern formation (Meinhardt, 1982; Murray, 1989; Meinhardt and Gierer, 2000). In fact, there is experimental evidence for the inclusion of quadratic terms. It seems that TTG lacks activation domains, unlike GL3 (Payne et al., 2000), WER (Lee and Schiefelbein, 1999) and GL 1 (Oppenheimer et al., 1991). Moreover, GL3 activity apparently depends on WER as the hairy phenotype on roots 
over-expressing $G L 3$ is suppressed when $W E R$ is non-functional (Bernhardt et al., 2003), while GL3 function does not depend on TTG (Payne et al., 2000; Bernhardt et al., 2003; Zhang et al., 2003). This data supports that in order to form functional multimers, at least functional GL3 (or a redundant protein, EGL3) and either GL1 or WER must be present According to this and to the mass-action law the quadratic nature of the activation terms $k_{2} \frac{a_{x, y}{ }^{2}}{i_{x, y}}$ and $k_{4} a_{x, y}^{2}$ follows.

To discard that differences in boundary conditions were the cause of differences in pattern we also modeled the root system using zero-flux boundary conditions in all borders (data not shown); the patterns were identical to those discussed above.

Equation 3 was modified to simulate constituve expression or WER, yielding

$$
\frac{\partial a_{x, y}}{\partial t}= \begin{cases}D_{a} \nabla^{2} a_{x, y}-k_{1} a_{x, y}+k_{2} \frac{a_{x, y}{ }^{2}}{i_{x, y}}+f(x)+W & \text { if }(x, y) \in N \\ D_{a} \nabla^{2} a_{x, y}-k_{1} a_{x, y} & \text { if }(x, y) \notin N\end{cases}
$$

where $W$ represents the constant production rate of WER protein simulating its production in 35S:WER transgenic plants.

In order to address if the patterns attained were sensitive to stochastic transient perturbations in the concentration of the molecules involved in the system, we repeated the simulations adding a stochastic term in equations (1) and (3) for both the leaf and root systems. Given that diverse patterns may arise when varying parameter values in Turing systems, we also tested deviations in single parameter values in order to test if results depend on a specific combination of parameter values (see Appendix). Equations were solved numerically using Euler's method (Burden and Faires, 1997) with a timestep equal to 0.01 or 0.001 . Both timestep values resulted in equivalent patterns. Programs were written in $\mathrm{C}_{++}$and are available upon request. Results were graphed using MATLAB.

\section{Acknowledgements}

We thank F. Sánchez-Garduño and E. Ortiz for their useful comments. Financial support was from Programa de Apoyo a Proyectos de Investigación e Innovación Tecnológica, Universidad NacionalAutónoma de México IN230002 and IX207104, University of California-MEXUS ECO IE 271 and Consejo Nacional de Ciencia y Tecnología CO1.41848/ A-1, CO1.0538/A-1 and CO1.0435.B-1 grants to E.A.B and Ph.D. scholarships from Consejo Nacional de Ciencia y Tecnología and Universidad Nacional Autónoma de México to C.E.-S and M.B. We thank R. V. PérezRuiz and $A$. Navarrete for technical and logistical assistance.

\section{Note added in proof}

A paper published while the proofs of this manuscript were being prepared, provides evidence that the gene SCRAMBLED(SCM)encodes a very important component of the biasing signal and that this inhibits the transcription of WER, which is a component of the activator complex

\section{TABLE 1}

\begin{tabular}{llcccc} 
Sign & Target & $\mathbf{0 . 0 6}$ & $\mathbf{0 . 3}$ & $\mathbf{0 . 6}$ & $\mathbf{6 . 0}$ \\
\hline+ & Activator & $\mathrm{Y}$ & $\mathrm{Y}$ & $\mathrm{Y}$ & $\mathrm{Y}$ \\
+ & Inhibitor & $\mathrm{N}$ & $\mathrm{N}$ & $\mathrm{N}$ & $\mathrm{N}$ \\
- (degradation) & Inhibitor & $\mathrm{N}$ & $\mathrm{N}$ & $\mathrm{Y}$ & $\mathrm{Y}$ \\
- (less production) & Inhibitor & $\mathrm{N}$ & $\mathrm{N}$ & $\mathrm{N}$ & $\mathrm{N}$ \\
- (degradation) & Activator & $\mathrm{N}$ & $\mathrm{N}$ & $\mathrm{N}$ & $\mathrm{N}$ \\
- (less production) & Activator & $\mathrm{N}$ & $\mathrm{N}$ & $\mathrm{N}$ & $\mathrm{N}$
\end{tabular}

$\mathrm{Y}$, simulations in which the root-like pattern was recovered; $\mathrm{N}$, simulations in which the root-like pattern was not recovered. All other parameters kept the same. We considered two kinds of negative signals; degradation of target proteins/mRNA or decreased production of target.
(Kwak \& Schiefelbein, 2006, Dev. Biol. In press). Our work suggests that, given relatively low biasing signal intensities (up to 0.3 ), root-like patterns arise only when the biasing signal acts positively on the activator. Hence, our prediction seems to conflict with the experimental evidence provided by Kwak \& Schiefelbein (2006). However, Kwak \& Schiefelbein (2006) also show that in the absence of $S C M$, the spatial root pattern of alternate atrichoblast and trichoblast columns is not lost completely. This suggests that there are additional components of the biasing signal which act through an SCM-independent pathway, and that the biasing signal is more complex than we had thought according to previous data. This is also supported by additional simulations (see Table 1 below, added during proof stage) which show that, given the assumptions of our model, root-like patterns are not recovered when the signal acts exclusively by repressing the activator, even with a 100 -fold increase in signal intensity. We are thus extending our model to enable explorations of the necessity and sufficiency of different types of biasing signals. Such analyses are only possible with a dynamic model as the one presented here.

\section{References}

ALBERT, R. and OTHMER, H. (2003). The topology of the regulatory interactions predicts the expression pattern of the segment polarity genes in Drosophila melanogaster. J. Theor. Biol. 223: 1-18.

BERGER, F., HUNG, C., DOLAN, L. and SCHIEFELBEIN, J. (1998). Control of cell division in the root epidermis of Arabidopsis thaliana. Dev. Biol. 194: 235-245.

BERNHARDT, C., LEE, M., GONZALEZ, A., ZHANG, F., LLOYD, A. and SCHIEFELBEIN, J. (2003). The bHLH genes GLABRA3 (GL3) and ENHANCER OF GLABRA3 (EGL3) specify epidermal cell fate in the Arabidopsis root. Development 130: 6431-6439.

BERNHARDT, C., ZHAO, M., GONZALEZ, A., LLOYD, A. and SCHIEFELBEIN, J. (2005). The bHLH genes GL3 and EGL3 participate in an intercellular regulatory circuit that controls cell patterning in the Arabidopsis root epidermis. Development 132: 291-298.

BURDEN, R. and FAIRES, J. (1997). Numerical Analysis. Brooks/Cole Publishing Co., Pacific Grove, CA, 6th edition.

CAMPANIONI, P. and NICK, P. (2005). Auxin-dependent cell division and cell elongation. 1-naphtaleneacetic acid and 2,4-Dichlorophenoxyacetic acid activate different pathways. Plant Physiol. 137: 939-948.

COSTA, S. and DOLAN, L. (2003). Epidermal patterning genes are active during embryogenesis in Arabidopsis. Development 130: 2893-2901.

DI CRISTINA, M., SESSA, G., DOLAN, L., LINSTEAD, P., BAIMA, S., RUBERTI, I. and MORELLI, G. (1996). The Arabidopsis Athb-10 (GLABRA2) is an HD-Zip protein required for regulation of root hair development. Plant J., 10: 393-402.

DOLAN, L. (1996). Pattern in the root epidermis: an interplay of diffusible signals and cellular geometry. Ann. Bot. 77: 547-553.

DOLAN, L., DUCKET, C., GRIERSON, C., LINSTEAD, P., SCHNEIDER, K., LAWSON, E., DEAN, C., POETHING, S. and ROBERTS, K. (1994). Clonal relationships and cell patterning in the root epidermis of Arabidopsis. Development 120: 2465-2474.

DOLAN, L. (2006). Positional information and mobile transcriptional regulators determine cell pattern in the Arabidopsis root epidermis. J Exp Bot. 57: 51-54.

ESPINOSA-SOTO, C., PADILLA-LONGORIA, P. and ALVAREZ-BUYLLA, E. (2004). A Gene Regulatory Network Model for Cell-Fate Determination during Arabidopsis thaliana Flower Development That Is Robust and Recovers Experimental Gene Expression Profiles. Plant Cel/16: 2923-2939.

FAGARD, M., DESNOS, T., DESPREZ, T., GOUBET, F., REFREGIER, G., MOUILLE, G., MCCANN, M., RAYON, C., VERNHETTES, S. and HOFTE, $H$. (2000). PROCUSTE1 encodes a cellulose synthase required for normal cell elongation specifically in roots and dark-grown hypocotyls of Arabidopsis. Plant Cel/12: 2409-2423.

GALWAY, M., MASUCCI, J., LLOYD, A., WALBOT, V., DAVIS, R. and SCHIEFELBEIN, J. (1994). The TTG gene is required to specify epidermal cell fate and cell patterning in the Arabidopsis root. Dev. Biol. 166: 740-754.

GAN, Y., R. KUMIMOTO, C. LIU, O. RATCLIFFE, H. YU and P. BROUN. (2006). GLABROUS INFLORESCENCE STEMS Modulates the Regulation by Gibber- 
ellins of Epidermal Differentiation and Shoot Maturation in Arabidopsis. Plant Cel/18: 1383-1395.

GOODWIN, B. (2001). How the leopard changed its spots. The evolution of complexity. Princeton Science Library, U.S.A., 2nd edition.

HUNG, C., LIN, Y., ZHANG, M., POLLOCK, S., MARKS, M. and SCHIEFELBEIN, J. (1998). A common position-dependent mechanism controls cell-type patterning and GLABRA2 regulation in the root and hypocotyl epidermis of Arabidopsis. Plant Physiol. 117: 73-84.

KAUFFMAN, S. (1969). Metabolic stability and epigenesis in randomly constructed genetic nets. J. Theor. Biol. 22: 437-467.

KAUFFMAN, S. (1996). At home in the Universe: The search for laws of complexity. Penguin books, England.

KIM, I. and ZAMBRYSKI, P. (2005). Cell-to-cell communication via plasmodesmata during Arabidopsis embryogenesis. Curr. Opin. Plant Biol. 8: 593-599.

KIRIK, V., SIMON, M., HUELSKAMP, M. and SCHIEFELBEIN, J. (2004). The ENHANCER OF TRY AND CPC1 gene acts redundantly with TRIPTYCHON and CAPRICE in trichome and root hair cell patterning in Arabidopsis. Dev. Biol. 268: 506-513.

KURATA, T., ISHIDA, T., KAWABATA-AWAI, C., NOGUCHI, M., HATTORI, S., SANO, R., NAGASAKA, R., TOMINAGA, R., KOSHINO-KIMURA, Y., KATO, et al. (2005). Cell-to-cell movement of the CAPRICE protein in Arabidopsis root epidermal cell differentiation. Development 132: 5387-5398.

KWAK, S.-H., SHEN, R. and SCHIEFELBEIN, J. (2005). Positional signaling mediated by a receptor-like kinase in Arabidopsis. Science 307: 1111-1113.

LARKIN, J., MARKS, M., NADEAU, J. and SACK, F. (1997). Epidermal cell fate and patterning in leaves. Plant Cel/9: 1109-1120.

LARKIN, J., OPPENHEIMER, D., POLLOCK, S. and MARKS, M. (1993). Arabidopsis GLABROUS1 gene requires downstream sequences for function. Plant $\mathrm{Ce} / / 5$ : 1739-1748.

LARKIN, J., YOUNG, N., PRIGGE, M. and MARKS, M. (1996). The control of trichome spacing and number in Arabidopsis. Development 122: 997-1005.

LE, J., VANDENBUSSCHE, F., VAN DER STRAETEN, D. and VERBELEN, J.-P. (2001). In the early response of Arabidopsis roots to ethylene cell elongation is upand down-regulated and uncoupled from differentiation. Plant Physiol. 125: 519-522.

LEE, M. and SCHIEFELBEIN, J. (1999). WEREWOLF, a MYB-related protein in Arabidopsis, is a position-dependent regulator of epidermal cell patterning. Cell, 99: 473-483.

LEE, M. and SCHIEFELBEIN, J. (2001). Developmentally distinct MYB genes encode functionally equivalent proteins in Arabidopsis. Development 128: 1539-1546.

LEE, M. and SCHIEFELBEIN, J. (2002). Cell Pattern in the Arabidopsis Root Epidermis Determined by Lateral Inhibition with Feedback. Plant Cel/14:611618.

MASUCCI, J. and SCHIEFELBEIN, J. (1996). Hormones act downstream of TTG and GL2 to promote root hair outgrowth during epidermis development in the Arabidopsis root. Plant Cel/8: 1505-17.

MEINHARDT, H. (1982). Models of biological pattern formation. Academic Press, London.

MEINHARDT, H. and GIERER, A. (2000). Pattern formation by local selfactivation and lateral inhibition. BioEssays 22: 753-760.

MENDOZA, L. and ALVAREZ-BUYLLA, E. (1998). Dynamics of the genetic regulatory network of Arabidopsis thaliana flower morphogenesis. J. Theor. Biol. 193: 307-319.

MENDOZA, L. and ALVAREZ-BUYLLA, E. (2000). Genetic regulation of root hair development in Arabidopsis thaliana: a network model. J. Theor. Biol. 204: 311-326.

MENDOZA, L., THIEFFRY, D. and ALVAREZ-BUYLLA, E. (1999). Genetic control of flower morphogenesis in Arabidopsis thaliana: a logical analysis. Bioinformatics 15: 593-606.

MEYEROWITZ, E. (1997). Genetic control of cell division patterns in developing plants. Cell, 88: 299-308.

MURRAY, J. (1989). Mathematical Biology. Springer, Berlin.

NIJHOUT, H. (2003). Gradients, diffusion and genes in pattern formation. In MULLER, G. and NEWMAN, S., editors, Origination of organismal form. Beyond the gene in developmental and evolutionary biology, pp. 165-182. MIT press, Cambridge, Massachusetts.

OPPENHEIMER, D., HERMAN, P., SIVAKUMARAN, S., ESCH, J. and MARKS, M. (1991). A myb gene required for leaf trichome differentiation in Arabidopsis is expressed in stipules. Cel/67: 483-493.

PAYNE, T., ZHANG, F. and LLOYD, A. (2000). GL3 encodes a bHLH protein that regulates trichome development in Arabidopsis through interaction with GL1 and TTG1. Genetics 156: 1349-1362.

PESCH, M. and HÜLSKAMP, M. (2004). Creating a two-dimensional pattern de novo during Arabidopsis trichome and root hair initiation. Curr. Opin. Genet. Devel. 14: 422-427.

RAMIREZ-PARRA, E., LOPEZ-MATAS, M., FRUNDT, C. and GUTIERREZ, C. (2004). Role of an atypical E2F transcription factor in the control of Arabidopsis cell growth and differentiation. Plant Cel/16: 2350-2363.

RERIE, W., FELDMAN, K. and MARKS, M. (1994). The GLABRA2 gene encodes a homeodomain protein required for normal trichome development in Arabidopsis. Genes Dev. 8: 1388-1399.

RYU, K., KANG, Y., PARK, Y., HWANG, I., SCHIEFELBEIN, J. and LEE, M. (2005). The WEREWOLF MYB protein directly regulates CAPRICE transcription during cell fate specification in the Arabidopsis root epidermis. Development 132: 4765-4775.

SCHELLMANN, S., SCHNITTGER, A., KIRIK, V., WADA, T., OKADA, K., BEERMANN, A., THUMFAHRT, J., JURGENS, G. and HÜLSKAMP, M. (2002). TRIPTYCHON and CAPRICE mediate lateral inhibition during trichome and root hair patterning in Arabidopsis. EMBO J. 21: 5036-5046.

SCHERES, B. (2001). Plant cell identity. The role of position and lineage. Plant Physiol. 125: 112-114.

SCHNITTGER, A., FOLKERS, U., SCHWAB, B., JURGENS, G. and HÜLSKAMP, M. (1999). Generation of a spacing pattern: the role of TRIPTYCHON in trichome patterning in Arabidopsis. Plant Cel/11: 1105-1116.

SERNA, L. (2005). Epidermal cell patterning and differentiation throughout the apical-basal axis of the seedling. J. Exp. Bot. 56: 1983-1989.

SZYMANSKI, D., JILK, R., POLLOCK, S. and MARKS, M. (1998). Control of GL2 expression in Arabidopsis leaves and trichomes. Development 125: 11611171.

THOMAS, R. (1991). Regulatory networks seen as asynchronous automata: A logical description. J. Theor. Biol. 153: 1-23.

TURING, A. (1952). The chemical basis of morphogenesis. Philos. Trans. R. SoC. Lond. B Biol. Sci. 237: 37-72.

VON DASSOW, G., MEIR, E., MUNRO, E. and ODELL, G. (2000). The segment polarity network is a robust developmental module. Nature 406: 188-193.

WADA, T., KURATA, T., TOMINAGA, R., KOSHINO-KIMURA, Y., TACHIBANA, T., GOTO, K., MARKS, M., SHIMURA, Y. and OKADA, K. (2002). Role of a positive regulator of root hair development, CAPRICE, in Arabidopsis root epidermal cell differentiation. Development 129: 5409-5419.

WADA, T., TACHIBANA, T., SHIMURA, Y. and OKADA, K. (1997). Epidermal cell differentiation in Arabidopsis determined by a Myb homolog, CPC. Science 277: 1113-1116.

ZHANG, F., GONZALEZ, A., ZHAO, M., PAYNE, C. and LLOYD, A. (2003). A network of redundant bHLH proteins functions in all TTG1-dependent pathways of Arabidopsis. Development 130: 4859-4869.

Received: 1st June 2006

Reviewed by Referees: 7th August 2006

Modified by Authors and Accepted for Publication: 19th October 2006

Published Online: 31st January 2007 


\section{Appendix}

\section{Analysis of the number and profiles of the steady states for both the complete network and the reduced system.}

\section{TABLE S1}

STATE OF THE ACTIVATION COMPLEX AS A DEPENDENT VARIABLE OF THE ACTIVATION STATE OF WER (IN ROOT) OR GL1 (IN LEAF), TTG, GL3 AND EGL3.

\begin{tabular}{lcccc}
\multicolumn{3}{c}{ Activator genes } & & \\
\hline WER/GL1 & TTG & GL3 & EGL3 & Activator complex \\
\hline 0 & $X$ & $X$ & $X$ & 0 \\
1,2 & 0 & 0,1 & $X$ & 0 \\
1,2 & 1 & 0 & 0 & 0 \\
1,2 & 0 & 2 & $X$ & 1 \\
1,2 & 1 & 0 & 1 & 1 \\
1,2 & 1 & 1 & $X$ & 1 \\
1,2 & 1 & 2 & $X$ & 2 \\
\hline
\end{tabular}

A

TABLE S2

\begin{tabular}{|c|c|c|c|c|c|}
\hline \multicolumn{3}{|c|}{ Initial condition } & \multicolumn{3}{|c|}{ Steady state } \\
\hline $\begin{array}{l}C P C \text { in root } \\
T R Y \text { in leaf }\end{array}$ & $\begin{array}{l}T R Y \text { in root } \\
C P C \text { in leaf }\end{array}$ & $\begin{array}{l}\text { Activator } \\
\text { complex }\end{array}$ & $\begin{array}{l}C P C \text { in root } \\
T R Y \text { in leaf }\end{array}$ & $\begin{array}{l}T R Y \text { in root } \\
C P C \text { in leaf }\end{array}$ & $\begin{array}{l}\text { Activator } \\
\text { complex }\end{array}$ \\
\hline 0 & $x$ & 1 & 1 & 1 & 1 \\
\hline 1 & 0,1 & 1 & 1 & 1 & 1 \\
\hline$x$ & $x$ & 0 & 0 & 0 & 0 \\
\hline 2 & $x$ & 1 & 0 & 0 & 0 \\
\hline$x$ & $x$ & 2 & 0 & 0 & 0 \\
\hline 1 & 2 & 1 & 0 & 0 & 0 \\
\hline
\end{tabular}

\section{B}

Initial condition

Activator complex Inhibitor complex
Steady state

Activator complex Inhibitor complex

\begin{tabular}{llll}
1 & 1 & 1 & 1 \\
0 & $\mathrm{x}$ & 0 & 0 \\
1 & 2 & 0 & 0 \\
1 & 0 & 0 & 0 \\
2 & $\mathrm{x}$ & 0 & 0 \\
\hline
\end{tabular}

Steady states for the complete network $(A)$ and for the reduced network of an activator and an inhibitor (B). In both cases, the initial conditions from which each steady state is attained are listed. The GL2"on" state corresponds with the activator complex being in a state different from 0 .
The reduced network is dynamically equivalent to the reactive terms of a classic activator-inhibitor system (see Fig. S1).

We compare both the equilibrium points for the well-mixed case (no diffusion) and the reduced network stationary states. In a typical activator-inhibitor system with no sources, there are two equilibria: the origin, which is unstable and another one, asymptotically stable (see for instance the standard reference by Murray (1989) on mathematical biology).

For fixed time intervals, a well known theorem (continuous dependence with respect to parameters and initial conditions) guarantees that a discretization can be made as accurate as possible. However, this is not the case when long times are involved. In our case, the scenario cannot be avoided, since the nontrivial equilibrium point is asymptotically stable and therefore acts as a global attractor. However, from the phase portrait, we can observe that for moderately long times and initial conditions not near this nontrivial equilibrium, the behavior of a system with a limit cycle and the behavior of the system with a stable spiral are very similar. In other words, when approximating a continuous dynamical system by a discrete one, there can be several possibilities, depending on the time-scale at which the system is to be approximated.

Another important issue here is that, if no limit cycle is introduced and one tries to reason in the opposite direction, that is, to recover the continuous dynamical system from the given discrete approximation, an spurious equilibrium point is introduced as implied by the Poincaré-Bendixon theorem.

Solving the simplified system is a useful approach that is further justified because:

1. If after solving the simplified system meaningful dynamics are recovered, this serves as a posteriorijustification of the fact that the reduction is valid.

2. Provided that the genes in the activator complex play a similar role (directly or indirectly), the reactive functions in a full model (including all of them) become functions depending, besides the inhibitor, on only one of the activator genes (the other two being functionally related). Therefore, the three reaction diffusion equations corresponding to each of the activator genes in the activator complex can be added to obtain an single equation for an "effective" activator. In other words, given that the three activator genes are highly correlated, the equation for any of them, or as just explained, an effective equation for the three can be used. 
A

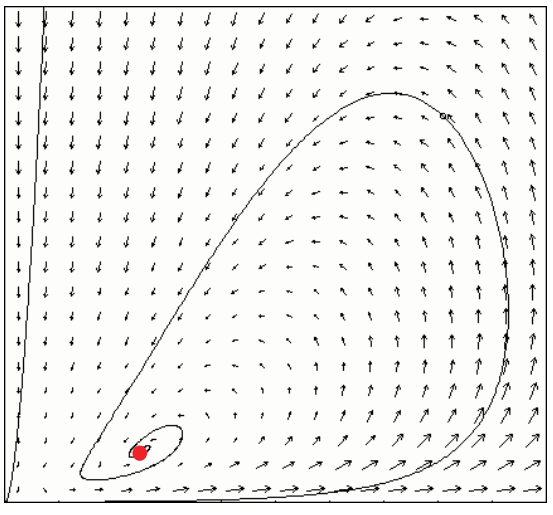

B

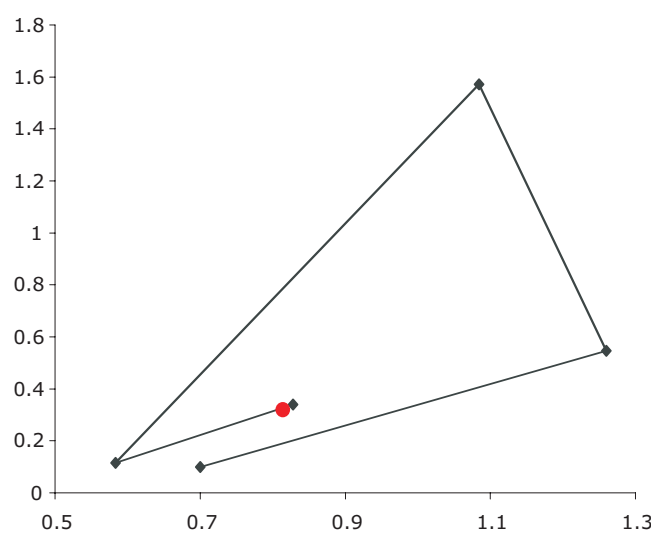

C

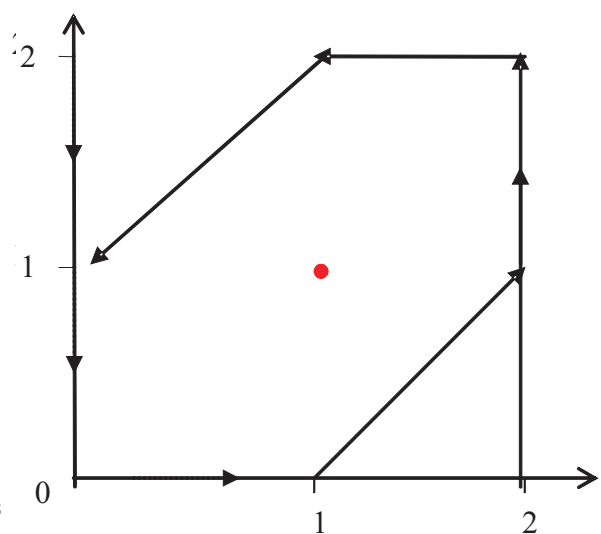

Fig. S1. Trajectories for the discrete reduced system (C) are qualitatively equivalent to those of the activator-inhibitor system (A) and to those obtained for a discretized activator-inhibitor system (B).

\section{Analysis of robustness of the model in the presence of transient perturbations and changes in the parameter values}

In order to test the robustness of the system in the presence of transient perturbations, we introduced a stochastic variable into equations 1 and 3 from the main text. The simulations were repeated for different intervals of this variable and in both cases, patterns equivalent to those obtained in the deterministic system were obtained for relatively strong noisy perturbations $(0.3)$.

We also addressed the question of whether our results depended on a specific combination of the parameter values. We found that this was not the case by picking random values of each parameter (see Table S3). The system's parameters may be associated to production, diffusion or degradation rates of the molecules involved. As shown in table S3, we found that the patterns obtained do not depend on particular or rare combinations of parameters. Both systems are considerably robust to alterations in parameter values, although the root system with elongated cells is more sensitive than the leaf one, especially regarding inhibitor's diffusion. However, it is not surprising that the root system with elongated cells is sensitive to the increase in this parameter since cell elongation mainly affects the communication among nuclei in the baso-apical direction. The leaf system is considerably more robust to parameter changes (results not shown).

TABLE S3

\begin{tabular}{ccccc}
\multicolumn{4}{c}{ PARAMETER (P) } \\
\hline $\begin{array}{c}\text { Inhibitor's } \\
\text { diffusion } \\
\text { coefficient }\end{array}$ & $\begin{array}{c}\text { Activator's } \\
\text { production }\end{array}$ & $\begin{array}{c}\text { Inhibitor's } \\
\text { production }\end{array}$ & $\begin{array}{c}\text { Activator's } \\
\text { degradation }\end{array}$ & $\begin{array}{c}\text { Inhibitor's } \\
\text { degradation }\end{array}$ \\
\hline
\end{tabular}

\begin{tabular}{lccccc}
\hline $\begin{array}{l}\text { Successful values } \\
(\%) \text { in }[P / 2, P]\end{array}$ & 28 & 100 & 4 & 72 & 4 \\
$\begin{array}{l}\text { Successful values } \\
(\%) \text { in }[\mathrm{P}, 2 \mathrm{P}]\end{array}$ & 0 & 36 & 96 & 4 & 2
\end{tabular}

The root system is robust when confronted with variations in the parameters. Each parameter $\left(D_{p}\right.$ $k_{1}, k_{2}, k_{3}$ and $k_{4}$ in equations 3 and 4 ) was varied randomly on a one-by-one basis, with a uniform distribution in the intervals $[P / 2, P]$ and $[P, 2 P][N=25$ for each interval], where $P$ is the parameter value for which simulations are shown in this investigation [Activator diffusion rate $\left(D_{2}\right)=0.15$; inhibitor diffusion rate $\left(D_{j}\right)=1.65$; activator degradation rate $\left(k_{t}\right)=0.3$; activator production constant $\left(k_{2}\right)=0.3$; inhibitor degradation rate $\left(k_{3}\right)=0.4$; inhibitor production constant $\left(k_{4}\right)=0.75$ ] The table presents the percentage of values for which the patterns resembling those in Arabidopsis are maintained. Parameters for the leaf system are more robust than those for the root system in the face of changes (results not shown).

\section{Stability analysis I: reactive part of the model for the nuclear interactions}

From the original set of equations (1) and (2) of the main text,

$$
\begin{aligned}
& \frac{\partial a}{\partial t}=-k_{1} a+k_{2} \frac{a^{2}}{i}+D_{a} \nabla^{2} a \\
& \frac{\partial i}{\partial t}=-k_{3} i+k_{4} a^{2}+D_{i} \nabla^{2} i
\end{aligned}
$$

we propose the following reassignment of variables in order to obtain an adimensional set of equations from the original system (S1):

$\tau=\frac{D_{i}}{L^{2}} t$

$a_{o} a=A$

$i_{o} i=I$

$u=\frac{x}{L}$

$v=\frac{y}{L}$

This set leads to

$$
\begin{aligned}
& \frac{\partial A}{\partial \tau}=\Psi\left(-\alpha A+\beta \frac{A^{2}}{I}\right)+\varepsilon\left[\frac{\partial^{2} A}{\partial u^{2}}+\frac{\partial^{2} A}{\partial v^{2}}\right] \\
& \frac{\partial I}{\partial \tau}=\Psi\left(-\gamma I+A^{2}\right)+\left[\frac{\partial^{2} I}{\partial u^{2}}+\frac{\partial^{2} I}{\partial v^{2}}\right]
\end{aligned}
$$

where 


$$
\begin{aligned}
& \Psi=\frac{L^{2} k_{2}}{D_{i}} \\
& \alpha=\frac{k_{1}}{k_{2}} \\
& \beta=\frac{i_{o}}{a_{o}} \\
& \gamma=\frac{k_{3}}{k_{2}} \\
& \frac{k_{4} i_{o}}{k_{2} a_{o}^{2}}=1 \\
& \varepsilon=\frac{D_{a}}{D_{i}}
\end{aligned}
$$

The steady state obtained from (S3) without the diffusive part is:

$$
A^{o}=\frac{\beta \gamma}{\alpha} \quad I^{o}=\frac{\beta^{2} \gamma}{\alpha^{2}}
$$

When the system (S3) is linearized in the neighborhood of the steady state (S5), assuming small perturbations $\delta A$ and " $\delta /$ from this value, the Jacobian matrix of the perturbed system is:

$$
\mathbf{J}=\Psi\left(\begin{array}{cc}
\alpha & -\frac{\alpha^{2}}{\beta} \\
\frac{2 \beta \gamma}{\alpha} & -\gamma
\end{array}\right)
$$

Using (S6), we can write the linearized reactive system as:

$$
\frac{\partial}{\partial \tau}\left[\begin{array}{l}
\delta A \\
\delta I
\end{array}\right]=\mathrm{J}\left[\begin{array}{l}
\delta A \\
\delta I
\end{array}\right]
$$

With the characteristic equation

$$
\lambda^{2}+\Psi(\gamma-\alpha) \lambda+\Psi^{2} \alpha \gamma=0
$$

the corresponding eigenvalues can be computed from:

$$
\lambda=\Psi\left[\frac{(\alpha-\gamma)}{2} \pm \frac{\sqrt{(\alpha-\gamma)^{2}-4 \alpha \gamma}}{2}\right]
$$

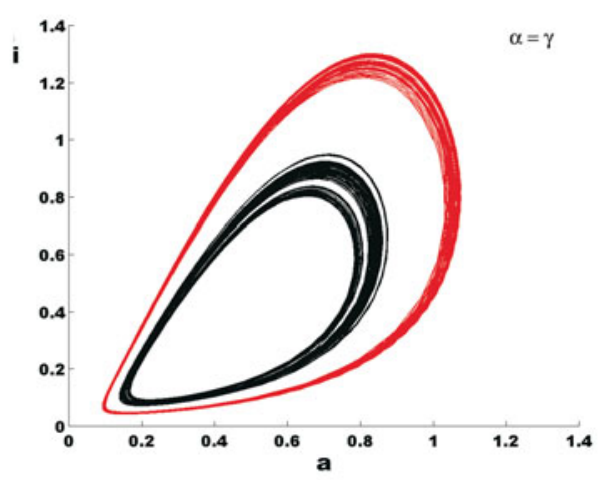

Fig. S2. In this figure: $\mathrm{k}_{1}=\mathrm{k}_{3}=0.4$, with $\mathrm{k}_{2}=0.3$ and $\mathrm{k}_{4}=0.75$ from the original system of equations (S1), which corresponds to $\alpha=\gamma=1.33$ in system (S3). The set of equations (S1) were numerically integrated using the Runge-Kutta 45 method, without any consideration being given to the diffusive part. $\Psi=1$ in both cycles. For the red curve, the initial conditions were $\mathrm{i}=1$ and $\mathrm{a}=1 ;$ for the black curve, the initial conditions were $\mathrm{i}=0.8$ and $\mathrm{a}=0.5$.

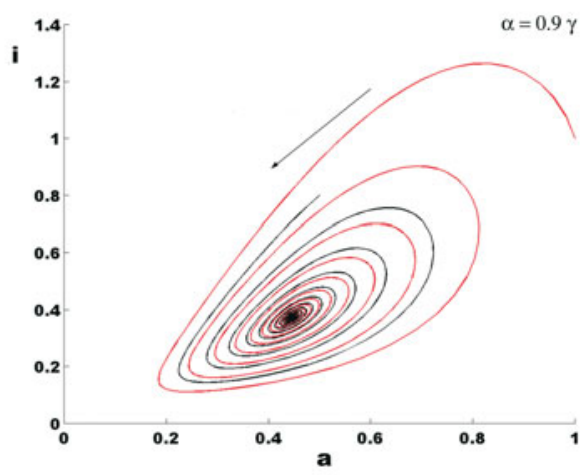

Fig. S3. Here, $\mathrm{k}_{1}=0.3591, \mathrm{k}_{2}=0.3 \mathrm{k}_{3}=0.4, \mathrm{k}_{4}=0.75$, which corresponds to $\alpha=0.9 y$ and $\Psi=1$. For the red curve, the initial conditions were $\mathrm{i}=1$ and $\mathrm{a}=1$ and for the black curve, the initial conditions were $\mathrm{i}=0.8$ and $\mathrm{a}=0.5$.

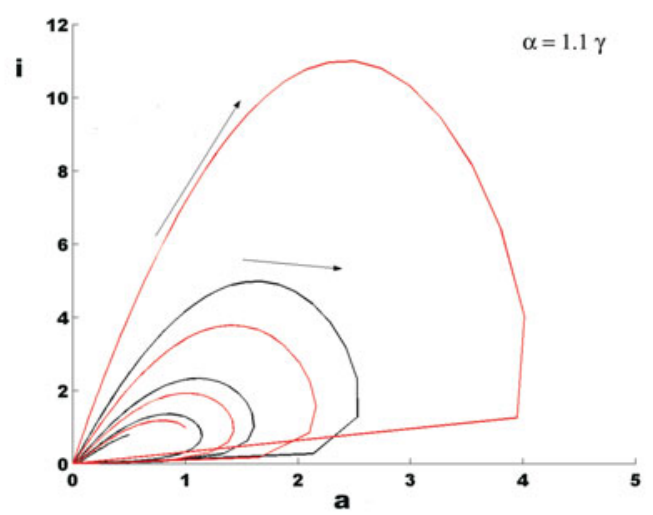

Fig. S4. In this figure, $\mathrm{k}_{1}=0.4389, \mathrm{k}_{2}=0.3 \mathrm{k}_{3}=0.4, \mathrm{k}_{4}=0.75$, which correspond to $\alpha=1.1 \gamma$ and $\Psi=1$. For the red curve, the initial conditions were $\mathrm{i}=1$ and $\mathrm{a}=1$ and for the black curve, the initial conditions were $\mathrm{i}$ $=0.8$ and $\mathrm{a}=0.5$. 
If we take $\gamma$ as the bifurcation parameter, we can characterize the temporal behavior of the system (S3) from the behavior of the eigenvalues computed from equation (S9). In this case, we have the following dynamical features of the system:

1) If $\alpha=\gamma$, then (S9) has two pure imaginary conjugated roots $\lambda=$ $\pm \Psi \gamma$ i. In this case the steady state point becomes a center and the system evolves into a cycle with angle velocity $\omega=\Psi \gamma$.

2) If $\alpha<\gamma$ and the discriminant part of (S9) is such that $\alpha^{2}-6 \alpha \gamma+$ $\gamma^{2}<0$, then (S8) has two conjugated complex roots with $\operatorname{Re}(\lambda)<$ 0 . This is true for all values $\alpha \in((3-2 \sqrt{2}) \gamma, \gamma)$. In this case the system evolves to a stable steady state through a spiral trajectory. 3) If $\alpha$ 's $\gamma$ and the discriminant part of (S9) is such that $\alpha^{2}-6 \alpha \gamma+$ $\gamma^{2}<0$, then (S8) has two conjugated complex roots with $\operatorname{Re}(\lambda)>$ 0 . This is true for all values $\alpha \in(\gamma,(3+2 \sqrt{2}) \gamma)$. In this case, the system escapes from the unstable steady state through a spiral trajectory.

4) If $\alpha<\gamma$ and the discriminant part of (S9) is such that $\alpha^{2}-6 \alpha \gamma+$ $\gamma^{2}>0$, then (S8) has two negative real roots. This is true for all values $\alpha \in(-\infty,(3-2 \sqrt{2}) \gamma)$. In this case, the steady state is stable.

5) If $\alpha>\gamma$ and the discriminant part of (S9) is such that $\alpha^{2}-6 \alpha \gamma+$ $\gamma^{2}>0$, then (S8) has two positive real roots. This is true for all values $\alpha \in((3+2 \sqrt{2}) \gamma, \infty)$. In this case, the steady state is unstable.

In Fig. S5, we present the bifurcation diagram corresponding to equation (S8). The bifurcation parameter is $\gamma$ and we use the linear relation $\alpha=b \gamma$, where $b$ takes the corresponding value indicated in the abscissa axis of the figure.

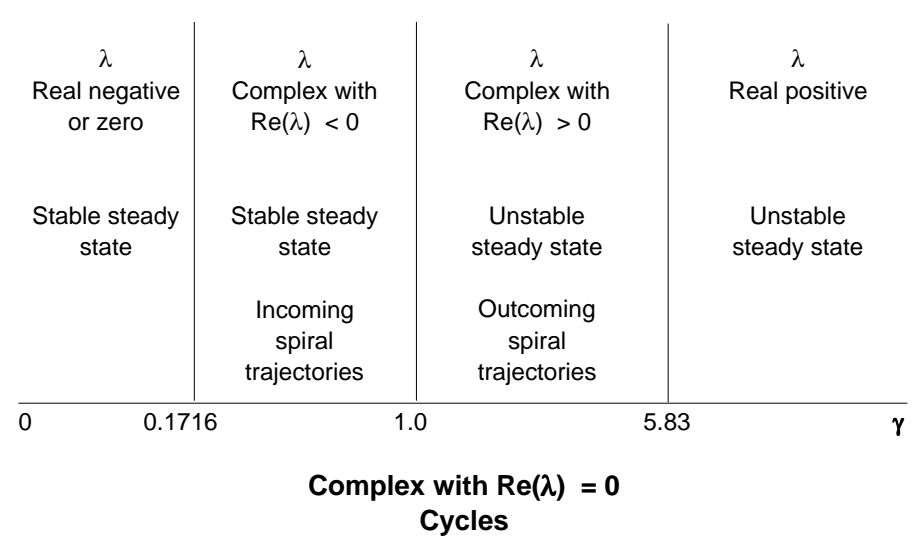

Fig. S5. Bifurcation diagram for the reactive part of the model.

\section{Stability analysis II: diffusive part of the model for the nuclear interactions}

If the system (S3) is now linearized around the steady state (S5), then in considering the diffusive component, we obtain the vector expression:

$$
\frac{d}{d \tau} \delta \mathbf{w}=\mathbf{J} \delta \mathbf{w}+\mathrm{n} \nabla^{2} \delta \mathbf{w}
$$

where:

$$
\delta \mathbf{w}=\left[\begin{array}{c}
\delta A \\
\delta I
\end{array}\right] \quad \mathrm{n}=\left(\begin{array}{cc}
\varepsilon & 0 \\
0 & 1
\end{array}\right)
$$

If we now define the operator: $\mathbf{H}=\mathbf{J}+\mathrm{n} \nabla^{2}$, then we have the following linear problem with boundary conditions:

$\frac{\partial}{\partial \tau} \delta \mathbf{w}=\mathbf{H} \delta \mathbf{w} \quad$ subject to $(\mathbf{n} \cdot \nabla) \delta \mathbf{w}=\mathbf{0}$ at the boundary

We now can propose as the solution to this linear problem the vectorial function given by

$\delta \mathbf{w}=\mathbf{C}_{\mathbf{l}, \mathbf{m}} e^{\lambda \tau} \cos \left(\frac{l \pi u}{p}\right) \cos \left(\frac{m \pi v}{q}\right)$

which leads to

$$
\begin{aligned}
& \mathbf{C}_{l, m} \cos \left(\frac{l \pi u}{p}\right) \cos \left(\frac{m \pi v}{q}\right) \lambda e^{\lambda \tau}=\mathbf{J} \mathbf{C}_{l, m} e^{\lambda \tau} \cos \left(\frac{l \pi u}{p}\right) \cos \left(\frac{m \pi v}{q}\right) \\
& -\mathrm{n} \mathbf{C}_{l, m} e^{\lambda \tau}\left[\frac{l^{2} \pi^{2}}{p^{2}} \cos \left(\frac{l \pi u}{p}\right) \cos \left(\frac{m \pi v}{q}\right)+\frac{m^{2} \pi^{2}}{q^{2}} \cos \left(\frac{l \pi u}{p}\right) \cos \left(\frac{m \pi v}{q}\right)\right]
\end{aligned}
$$

and

$$
\begin{aligned}
& \mathbf{C}_{l, m} \cos \left(\frac{l \pi u}{p}\right) \cos \left(\frac{m \pi v}{q}\right) \lambda-\mathbf{J C}_{l, m} \cos \left(\frac{l \pi u}{p}\right) \cos \left(\frac{m \pi v}{q}\right)= \\
& -\mathrm{nC}_{l, m}\left[\frac{l^{2} \pi^{2}}{p^{2}} \cos \left(\frac{l \pi u}{p}\right) \cos \left(\frac{m \pi v}{q}\right)+\frac{m^{2} \pi^{2}}{q^{2}} \cos \left(\frac{l \pi u}{p}\right) \cos \left(\frac{m \pi v}{q}\right)\right]
\end{aligned}
$$

Finally,

$\lambda \mathbf{I}-\mathbf{J}=-\mathrm{n}\left[\frac{l^{2} \pi^{2}}{p^{2}}+\frac{m^{2} \pi^{2}}{q^{2}}\right]$

where

$\kappa^{2}=\pi^{2}\left(\frac{l^{2}}{p^{2}}+\frac{m^{2}}{q^{2}}\right)$ 
and

$\mathbf{J}-\lambda \mathbf{I}-\mathrm{n} \kappa^{2}=0$

Thus:

$$
\delta \mathbf{w}(u, v, \tau)=\sum_{j} \sum_{l} \sum_{m} \mathbf{C}_{l, m} e^{\lambda_{j}\left(\kappa^{2}\right) \tau} \cos \left(\frac{l \pi u}{p}\right) \cos \left(\frac{m \pi v}{q}\right)
$$

The characteristic equation of the non-diffusive part of the linearized system has two eigenvalues, thus the solution to equation (S12), subject to the given boundary conditions, indicates that $j=$ 1,$2 ; /=0, \pm 1, \pm 2, \pm 3, \ldots ; m=0, \pm 1, \pm 2, \pm 3, \ldots$, with $p=q=1$ and that the system wave number is given by (S17).

Based on (S12) to (S19), we can give the secular equation of the linearized system as:

$$
\operatorname{det}\left(\mathbf{J}-\mathrm{n} \kappa^{2}-\lambda \mathbf{I}\right)=0
$$

Taking the following values from the main text:

$k_{1}=0.3, k_{2}=0.3, k_{3}=0.4, k_{4}=0.75, L=1.5 ; D_{i}=1.65 ; D_{a}=0.15$,

we get: $\psi=0.41, \alpha=1, \gamma=1.333, \varepsilon=0.09$

Substituting these values into equation (S20), we find that there is no set of values of $\kappa^{2}$ for which there are eigenvalues with a real positive part. Thus, the system is stable and the probability of pattern formation is very small near the steady state.

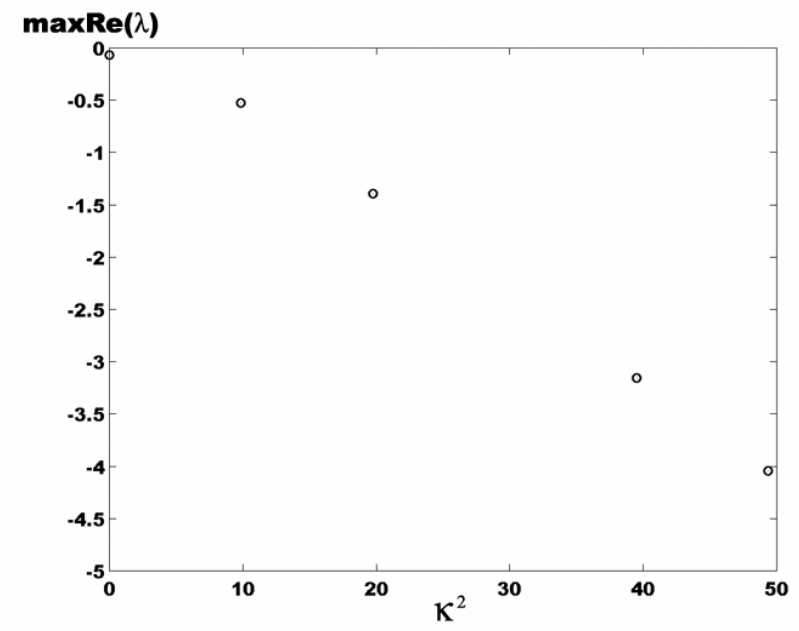

Fig. S6. In the absence of a biasing signal, all eigenvalues of equation (S18) have a negative real part, indicating that the steady state is stable against any perturbation and that spatial patterns may not arise for any value of $\mathrm{k}^{2}$ with $\mathrm{L}=1.5$

However, if the size of the system is increased to $L=10$, which leads to $\Psi=18.18$, there is a set of $\kappa^{2}$ values for which there are eigenvalues with a real positive part. The maximum value is obtained when $\kappa^{2} \sim 80$ (see Fig. S7).

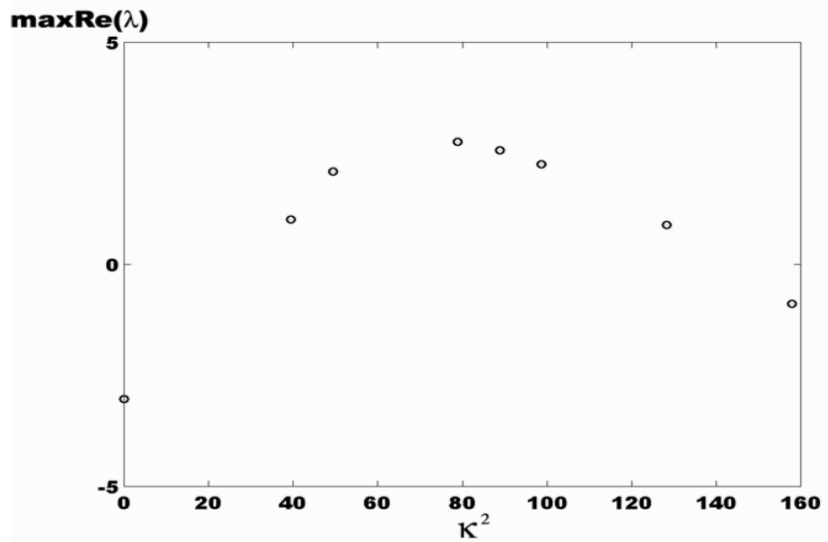

Fig. S7. Real parts of the eigenvalues of equation (S18) as a function of the wave number $\mathrm{K}^{2}$ for the cells in the absence of a biasing signal and for $\mathrm{L}=10$.

This value of $\kappa^{2}$ corresponds to the mode $/=2$ and $m=2$. The expected type of spatial pattern for this value of $\kappa^{2}$ is of the form:

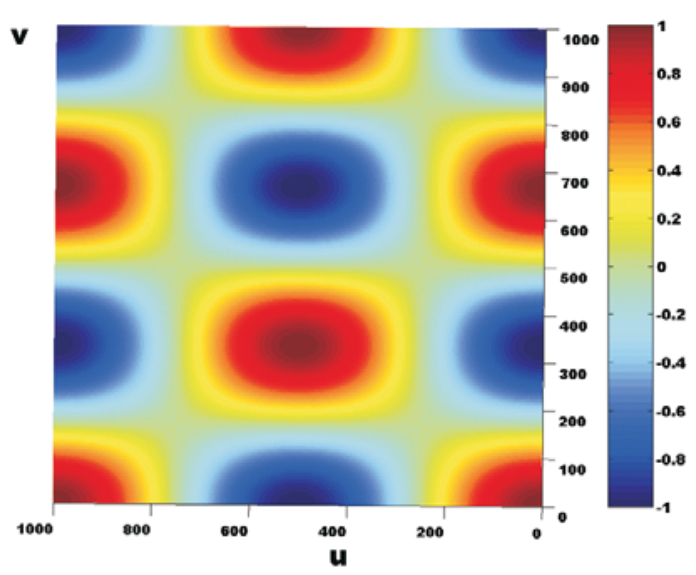

Fig. S8. Expected dissipative spatial pattern expected for the maximum positive real part of the eigenvalue corresponding to $\kappa^{2} \sim 80$. In this case, the modes are $I=2$ and $m=2$.

As we increase $L$, the value of $\psi$ increases, fixing the values of $k_{2}$ and $D_{i}$. When $\Psi$ is $\sim 100$, which corresponds to a size of $\sim 29$, the maximum real positive part of the eigenvalues of equation (S18) is obtained when $\kappa^{2}=404$, which corresponds to the mode numbers: $/=5$ and $m=4$. The spatial pattern expected in this case is: 


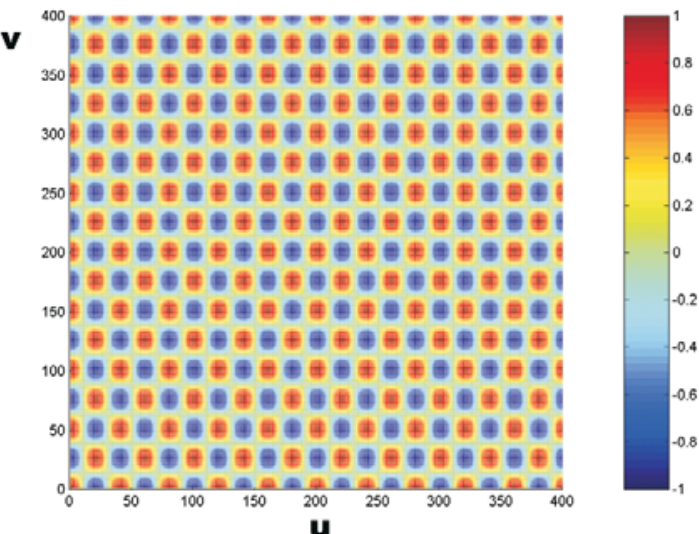

Fig. S9. Expected dissipative spatial pattern for the maximum positive real part of the eigenvalue corresponding to $\mathrm{K}^{2} \sim 404$. In this case, the modes are $\mathrm{I}=5$ and $\mathrm{m}=4 . \mathrm{L}=29$.

\section{CONCLUSION}

From this stability analysis we may conclude that it is quite unlikely that banded root-like patterns arise in the absence of a biasing signal similar to the one coming from the cortex cells towards the root epidermis. Therefore, such a biasing signal $f(x)$ seems to be not only sufficient but also necessary for the formation of the striped pattern of hair and non-hair cells in the root epidermis. 